\title{
Male offspring born to mildly ZIKV-infected mice are at risk of developing neurocognitive disorders in adulthood
}

\section{Stanelle-Bertram, Stephanie}

2018-10

Stanelle-Bertram , S , Walendy-Gnirss , K, Speiseder , T, Thiele , S , Asante , I A , Dreier , C , Kouassi , N M , Preuss , A, Pilnitz-Stolze , G, Mueller , U , Thanisch , S , Richter , M , Scharrenberg , R, Kraus , V , Doerk , R, Schau , L, Herder , V, Gerhauser , I, Pfankuche , V M , Kaeufer , C , Walt , I, Moraes , T, Sellau , J , Hoenow , S , Schmidt-Chanasit, J , Jansen , S , Schattling , B , Ittrich , H , Bartsch , U , Renne , T , Bartenschlager , R , Arck , P , Cadar , D , Friese , M A , Vapalahti , O , Lotter , H , Benites , S , Rolling , L , Gabriel , M , Baumgaertner , W , Morellini , F , Hoelter , S M , Amarie , O , Fuchs , H , de Angelis , M H, Loescher, W, de Anda, F C \& Gabriel , G 2018 , ' Male offspring born to mildly ZIKV-infected mice are at risk of developing neurocognitive disorders in adulthood ', Nature Microbiology , vol. 3 , no. 10 , pp. 1161-+ . https://doi.org/10.1038/s41564-018-0236-1

http://hdl.handle.net/10138/306618

https://doi.org/10.1038/s41564-018-0236-1

publishedVersion

Downloaded from Helda, University of Helsinki institutional repository.

This is an electronic reprint of the original article.

This reprint may differ from the original in pagination and typographic detail.

Please cite the original version. 


\title{
Male offspring born to mildly ZIKV-infected mice are at risk of developing neurocognitive disorders in adulthood
}

\author{
Stephanie Stanelle-Bertram', Kerstin Walendy-Gnirß1, Thomas Speiseder', Swantje Thiele', \\ Ivy Asantewaa Asante', Carola Dreier ${ }^{1}$, Nancy Mounogou Kouassi' ${ }^{1}$, AnnettePreuß ${ }^{1}$, Gundula \\ Pilnitz-Stolze', Ursula Müller', Stefanie Thanisch', Melanie Richter ${ }^{2}$, Robin Scharrenberg ${ }^{2}$, \\ Vanessa Kraus ${ }^{3}$, Ronja Dörk³ , Lynn Schau ${ }^{3}$, Vanessa Herder 4,5, Ingo Gerhauser (D) ${ }^{4}$ \\ Vanessa Maria Pfankuche ${ }^{4,5}$, Christopher Käufer ${ }^{6}{ }^{6}$, Inken Waltl ${ }^{6}$, Thais Moraes ${ }^{7}$, JulieSellau ${ }^{8}$, \\ Stefan Hoenow ${ }^{8}$, Jonas Schmidt-Chanasit ${ }^{8,9}$, Stephanie Jansen ${ }^{8}$, Benjamin Schattling ${ }^{10}$, Harald Ittrich ${ }^{11}$, \\ UdoBartsch $^{12}$, Thomas Renné ${ }^{13}$, RalfBartenschlager ${ }^{7}{ }^{7}$, Petra Arck $^{14}$, Daniel Cadar ${ }^{8}$, Manuel A. Friese ${ }^{10}{ }^{10}$, \\ Olli Vapalahti ${ }^{15}$, Hanna Lotter ${ }^{8}$, Sany Benites ${ }^{16}$, Lane Rolling ${ }^{16}$, Martin Gabriel ${ }^{8}$, Wolfgang Baumgärtner ${ }^{4,5}$, \\ Fabio Morelliniं ${ }^{3}$, SabineM. Hölter (17),18, Oana Amarie ${ }^{17,18}$, Helmut Fuchs ${ }^{18}$, Martin Hrabe de Angelis ${ }^{18,19,20}$, \\ Wolfgang Löscher ${ }^{6}$, Froylan Calderon de Anda ${ }^{2}$ and Gülsah Gabriel $\mathbb{1}^{1,9,21,22 \star ~}$
}

Congenital Zika virus (ZIKV) syndrome may cause fetal microcephaly in $\sim 1 \%$ of affected newborns. Here, we investigate whether the majority of clinically inapparent newborns might suffer from long-term health impairments not readily visible at birth. Infection of immunocompetent pregnant mice with high-dose ZIKV caused severe offspring phenotypes, such as fetal death, as expected. By contrast, low-dose (LD) maternal ZIKV infection resulted in reduced fetal birth weight but no other obvious phenotypes. Male offspring born to LD ZIKV-infected mothers had increased testosterone (TST) levels and were less likely to survive in utero infection compared to their female littermates. Males also presented an increased number of immature neurons in apical and basal hippocampal dendrites, while female offspring had immature neurons in basal dendrites only. Moreover, male offspring with high but not very high (storm) TST levels were more likely to suffer from learning and memory impairments compared to females. Future studies are required to understand the impact of TST on neuropathological and neurocognitive impairments in later life. In summary, increased sex-specific vigilance is required in countries with high ZIKV prevalence, where impaired neurodevelopment may be camouflaged by a healthy appearance at birth.

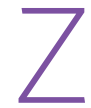
ika virus (ZIKV) was first isolated 1947 from sentinel Rhesus primates in Uganda. It is transmitted to humans via Aedes mosquitoes and may cause mild febrile disease accompanied by a maculopapular rash ${ }^{1}$. Since 2007 , ZIKV has spread from Micronesia to French Polynesia in 2013 and later to Brazil in 2015, causing several outbreaks. ZIKV strains cluster into an African and an Asian lineage, with the latter representing the current epidemic lineage ${ }^{2,3}$. In 2015, maternal ZIKV infection during pregnancy was associated with fetal loss, spontaneous abortions and neurological disorders, such as microcephaly in neonates ${ }^{4-6}$. Retrospective studies estimate that ZIKV-associated microcephaly is a very rare phenomenon. In Polynesia, the estimated risk of microcephaly due to

\footnotetext{
${ }^{1}$ Heinrich Pette Institute, Leibniz Institute for Experimental Virology, Hamburg, Germany. ${ }^{2}$ Neuronal Development, Center for Molecular Neurobiology Hamburg (ZNMH), University Medical Center Hamburg-Eppendorf, Hamburg, Germany. ${ }^{3}$ Research Group for Behavioral Biology, Center for Molecular

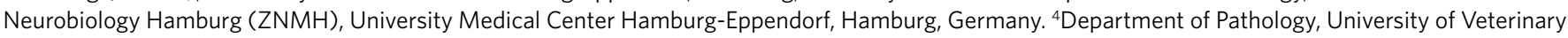
Medicine, Hannover, Germany. ${ }^{5}$ Center of Systems Neuroscience, Hannover, Germany. ${ }^{6}$ Department of Pharmacology, Toxicology and Pharmacy, University of Veterinary Medicine, Hannover, Germany. ${ }^{7}$ Department of Infectious Diseases, Molecular Virology, Heidelberg University, Heidelberg, Germany. ${ }^{8}$ Bernhard Nocht Institute for Tropical Medicine, Hamburg, Germany. ${ }^{9}$ German Centre for Infection Research (DZIF), Partner Site HamburgLübeck-Borstel-Riems, Hamburg, Germany. ${ }^{10}$ Institute of Neuroimmunology and Multiple Sclerosis, University Medical Center Hamburg-Eppendorf, Hamburg, Germany. "Department of Diagnostic and Interventional Radiology and Nuclear Medicine, University Medical Center Hamburg-Eppendorf, Hamburg, Germany. ${ }^{12}$ Department of Ophthalmology, University Medical Center Hamburg-Eppendorf, Hamburg, Germany. ${ }^{13}$ Institute for Clinical and Laboratory Chemistry, University Medical Center Hamburg-Eppendorf, Hamburg, Germany. ${ }^{14}$ Department of Obstetrics and Fetal Medicine, University Medical Center Hamburg-Eppendorf, Hamburg, Germany. ${ }^{15}$ University of Helsinki and Helsinki University Hospital, Helsinki, Finland. ${ }^{16}$ Tropical Pathology and Infectious Disease Association, Cusco, Peru. ${ }^{17}$ Institute of Developmental Genetics, Helmholtz Zentrum München, Neuherberg, Germany. ${ }^{18}$ German Mouse Clinic, Institute of Experimental Genetics, Helmholtz Zentrum München, Neuherberg, Germany. ${ }^{19}$ Chair of Experimental Genetics, School of Life Science Weihenstephan, Technical University of Munich, Freising, Germany. ${ }^{20}$ German Center for Diabetes Research (DZD), Neuherberg, Germany. ${ }^{21}$ Center for Structural and Cell Biology in Medicine, University of Lübeck, Lübeck, Germany. ${ }^{22}$ University of Veterinary Medicine, Hannover, Germany. *e-mail: guelsah.gabriel@leibniz-hpi.de
} 
ZIKV infection in the first trimester of pregnancy is $0.95 \%$, whereas in Brazil, it varies between 0.8 and $13.2 \%$, if infection occurs particularly during the first trimester ${ }^{7}$. However, recent clinical studies including a larger cohort size also report serious adverse outcomes until the third trimester on symptomatic maternal ZIKV infection ${ }^{6}$. Most importantly, speculations are growing that those children who do not present clinical manifestations at birth might suffer from health impairments in later life ${ }^{8}$. However, the long-term developmental trajectory for these clinically asymptomatic children, who represent the majority of newborns to ZIKV-infected mothers, remains currently unknown ${ }^{8}$.

To date, severe and fatal birth defects due to ZIKV infection were reported in a series of murine pregnancy models, including systemically infected immunodeficient ${ }^{9,10}$ or locally infected immunocompetent $^{11,12}$ mice. However, a fully immunocompetent pregnancy model of systemic ZIKV infection, which would allow a longitudinal monitoring of vital and clinically inapparent offspring with potential predictive value for human ZIKV infection, is still missing.

In this study, we sought evidence for the urgent medical question in the field whether 'healthy'-appearing offspring born to ZIKVinfected mothers, which represent the majority of newborns, might suffer from long-term health impairments ${ }^{8}$.

\section{Results}

We systemically infected immunocompetent pregnant mice ${ }^{13}$ at early gestation using a low-dose (LD) or as a control a high-dose (HD) 2016 ZIKV (Supplementary Fig. 1). Pregnant mice infected either with LD or HD 2016 ZIKV showed mild but significant differences in gestational weight gain compared to phosphate-buffered saline (PBS)-treated dams (Fig. 1a). To exclude that impaired maternal weight gain might be due to viral infection in general, we used dengue virus type 2 (DENV2) as a non-related flavivirus control. Maternal DENV2 infection did not affect maternal weight gain (Supplementary Fig. 2a-c), suggesting that ZIKV infection is specifically responsible for the detected impairment in gestational weight. However, to see whether the observed effects are further specific for the 2016 ZIKV outbreak strain, we used the historical MR766 strain as an additional control (herein referred to as 1947 ZIKV), where no maternal or fetal abnormalities were reported in humans before. Maternal 1947 ZIKV infection did not result in significant alterations in gestational weight similar to the infection courses in non-pregnant mice (Supplementary Fig. 2d,e). The proposed increased replicative fitness of the 2016 ZIKV strain compared to the 1947 ZIKV strain was further confirmed in human cells (Supplementary Fig. 2f). All 2016 ZIKV-infected pregnant mice seroconverted, presenting immunoglobulin $\mathrm{M}$ (IgM) and IgG antibodies (Supplementary Table 1), thus confirming successful viral infection. The $2016 \mathrm{ZIKV}$-infected dams showed elevated serum levels of the inflammation markers interleukin 6 (IL-6) and monocyte chemoattractant protein 1 (MCP-1) (Fig. 1b,c) correlating with peak systemic vRNA titres on day 1 post-infection (Supplementary Fig. 2g). However, IL-17A levels, a key marker of maternal immune activation (MIA) involved in fetal neurodevelopment on maternal poly(I:C) treatment ${ }^{14}$, were not altered (Fig. 1d). These findings suggest that maternal ZIKV infection triggers alternative pathways that are not IL-17A- but probably IL-6-dependent, which is another major factor elevated during MIA involved in fetal neurodevelopment ${ }^{15}$.

Sex hormone levels are altered on neurotropic maternal 2016 ZIKV infection. Maternal sex steroids play a key role in reproductive outcome and fetal neurodevelopment ${ }^{16}$. HD ZIKV infection resulted in significantly increased progesterone and reduced testosterone (TST) levels unlike oestradiol levels on day 6 postinfection compared to PBS-treated groups (Fig. 1e,f; Supplementary Fig. 2h). LD ZIKV infection resulted in elevated TST levels com- pared to controls (Fig. 1f). Progesterone modulates the synthesis and release of angiogenic factors by placental and decidual cells, which in turn may promote the invasion of trophoblast cells and affect uterine artery remodelling ${ }^{17}$. TST is involved in fetal programming, including neurodevelopment, particularly if hormonal alterations occur during early gestation ${ }^{18}$. Corticosterone levels, commonly used to assess prenatal stress ${ }^{19}$, were not significantly altered (Supplementary Fig. 2i). Interestingly, maternal infection severity during pregnancy correlated with a significant increase in spleen weight in 2016 ZIKV-infected pregnant and non-pregnant mice compared to PBS controls (Supplementary Fig. 2j). Thus, an altered expression pattern of steroid hormones, particularly of progesterone and TST, could be detected in ZIKV-infected mothers at early gestation. Next, we analysed whether the 2016 ZIKV strain isolated from fetal brain also replicates in the maternal murine adult brain since no information was available from the affected human case $^{2}$. In non-pregnant mice, among LD ZIKV-infected animals, one out of five tested presented ZIKV RNA in their brains on day 3 post-infection and 3/5 were ZIKV-positive on day 6 post-infection (Supplementary Table 2). In HD ZIKV-infected mice, all tested animals (5/5) were ZIKV RNA-positive on days 3 and 6 post-infection. Similarly, in pregnant mice, ZIKV RNA was detected in LD- and HD-infected dams, albeit the level of virus-positive probes was higher in the HD-infected maternal brains at 3, 6 and 14 days postinfection (Supplementary Table 2). Consistently, virus titres in the maternal brain were highest in the HD ZIKV group compared to the LD group on days 3 and 6 post-infection (Fig. 1g). Particularly glial cells, such as astrocytes, oligodendrocytes and microglia, were ZIKV-positive in the maternal brain (most profoundly in the brainstem and cerebellum; Fig. 1h). The 2016 ZIKV strain productively infected human neuronal progenitor cells (Fig. 1i), as reported previously ${ }^{20}$, further supporting the relevance of our murine model in mimicking clinical observations.

Mother-to-fetus $2016 \mathrm{ZIKV}$ transmission is associated with placental insufficiency. To address the question whether the 2016 ZIKV strain transmits from the mother to the fetus, we removed gravid and non-gravid uteri at 6.5 days gestation of ZIKV-infected dams and analysed them for the presence of viral RNA. Uteri from non-pregnant infected mice were in general ZIKV RNA-positive in the LD (4/5) and HD (5/5) groups at days 3 and 6 post-infection (Supplementary Table 2). In pregnant mice, at 3 days post-infection all analysed LD (6/6) and HD (7/7) uteri tested ZIKV-positive. On day 6 post-infection, 3/5 LD and 5/5 HD uteri were virus-positive (Supplementary Table 2). ZIKV RNA levels were higher on day 3 post-infection in the LD ZIKV pregnant compared to non-pregnant infected animals (Fig. 2a). Implantation sites were highly ZIKV RNA-positive on days 3 and 6 post-infection in both the LD (26/28 and 9/27, respectively) and HD (60/69 and 34/40, respectively) groups (Supplementary Table 2). Consistently, ZIKV titres were higher in LD compared to HD implants (Fig. 2b). Haemorrhagic placentation sites in uteri were particularly severe in the HD ZIKV group (Fig. 2c). Furthermore, significantly increased necrosis in the ZIKV-infected implantation sites was detected (Supplementary Fig. 2k,l). Decidual, placental and fetal epithelial and stroma cells at the implantation site were abundantly ZIKV RNA-positive at E6.5, a stage when the fetal neuroepithelium is already present. Viruspositive cells were most abundant at the interface between embryo and maternal tissue, including trophoblast giant cells and decidual cells (Fig. 2d; Supplementary Fig. 2m). The number of ZIKV RNApositive fetal cells increased with the maternal ZIKV infection dose as expected (Fig. 2d). Fetuses presented IgM titres in their sera on maternal LD and HD ZIKV infection at birth and adolescence (Supplementary Table 1). Since IgM antibodies do not cross the placenta, fetal IgM titres are indicative of successful fetal infection in utero, which is consistent with ZIKV positivity in fetal epithelia 

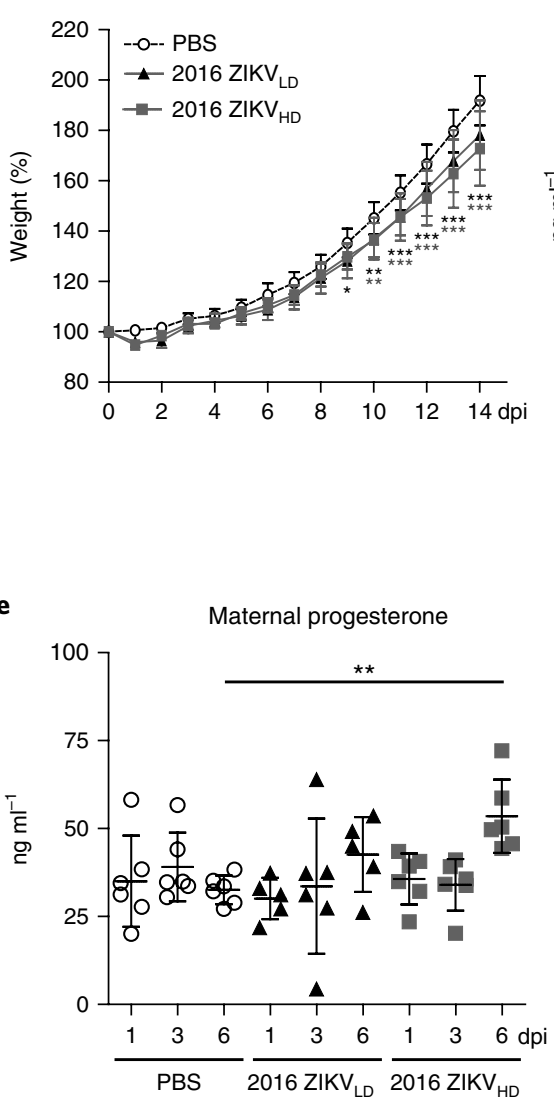

b

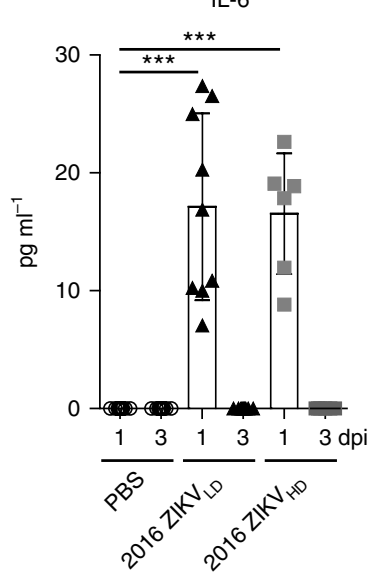

c

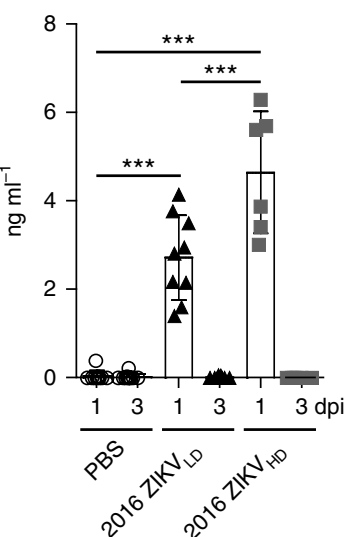

IL-17A

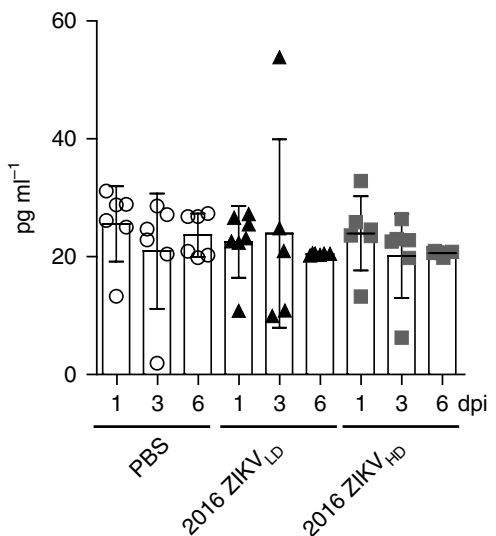

f

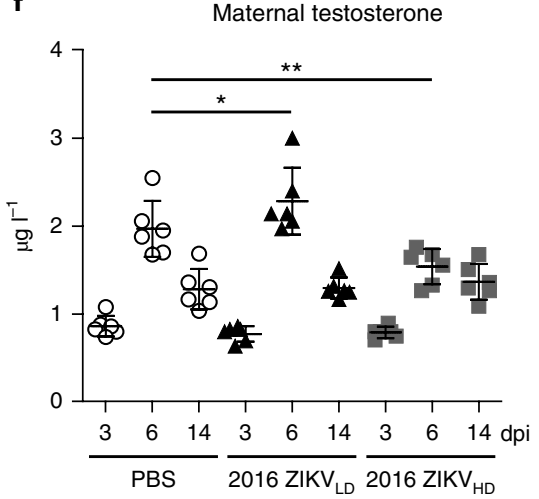

g

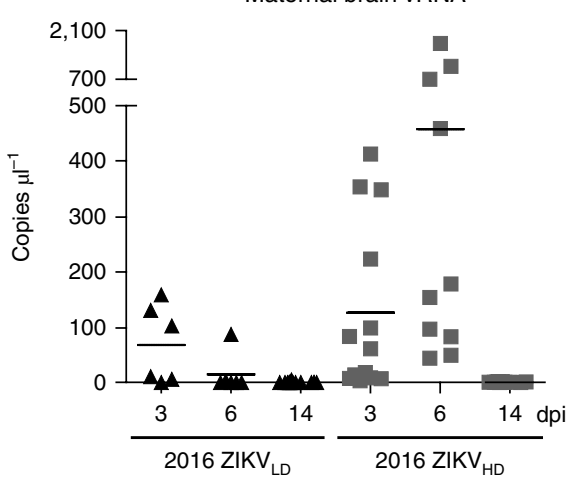

h

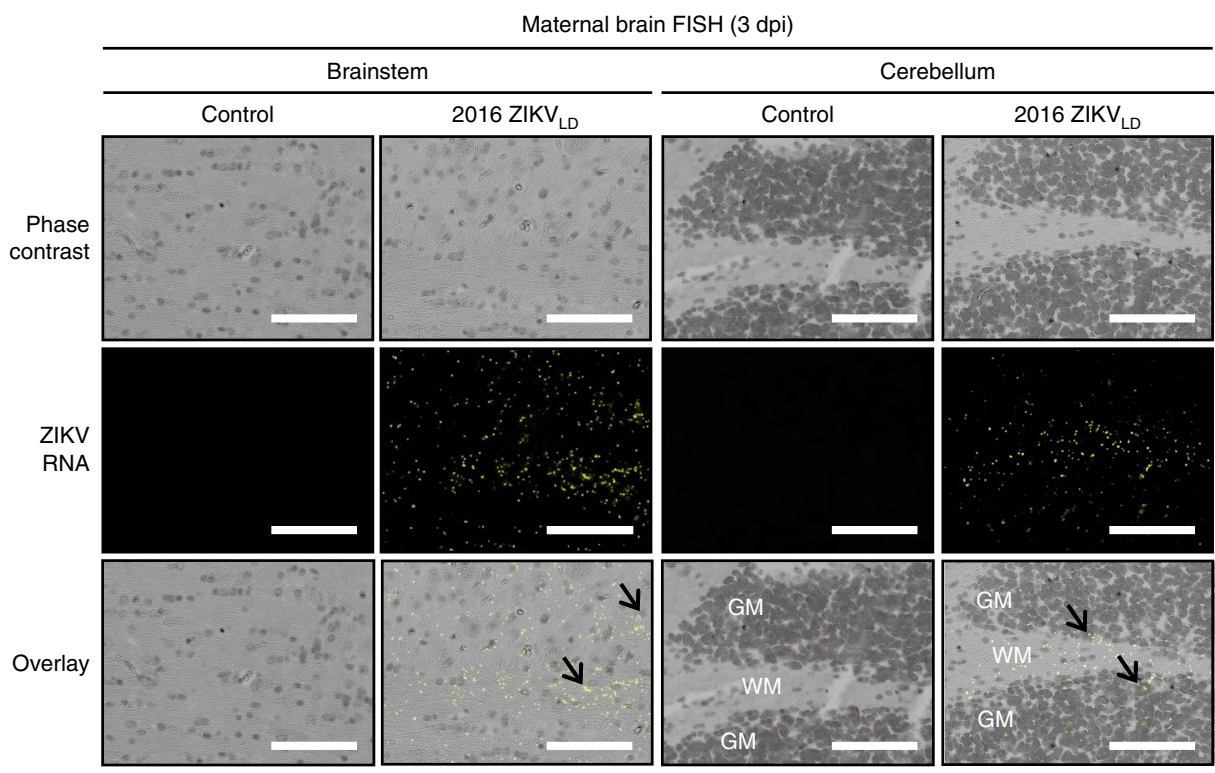

i

Virus replication NPC

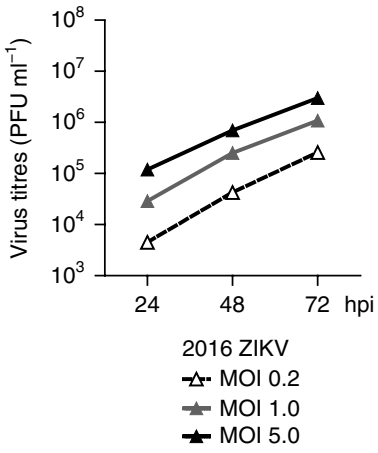

Fig. 1 | Maternal ZIKV pathogenesis in immunocompetent allogeneic pregnant mice. a, Weight gain of pregnant mice infected with $Z I K V\left(2016\right.$ ZIKV ${ }_{L D}: n=25$; 2016 ZIKV $_{\mathrm{HD}}: n=17$ ) or PBS (PBS: $\left.n=18\right)$. b-d, Cytokines IL-6 (b; PBS and 2016 ZIKV $\mathrm{LD}: n=9 ; 2016$ ZIKV $\mathrm{HD}: n=6$ ), MCP-1 (c, also known as CCL2, PBS and $\left.2016 \mathrm{ZIKV}_{\mathrm{LD}}: n=9 ; 2016 \mathrm{ZIKV}_{\mathrm{HD}}: n=6\right)$ and IL-17A $(\mathbf{d} ; n=6)$ detected in maternal serum. e,f, Progesterone (e) and TST (f) levels detected in maternal serum (PBS: $n=6 ; 2016 \mathrm{ZIKV}_{\mathrm{LD}}: n=5$ for progesterone and $n=6$ for TST; $2016 \mathrm{ZIKV}_{\mathrm{HD}}: n=6$ ). (a-f, Values are shown as means, error bars as s.d., two-way ANOVA, Bonferroni post hoc tests, ${ }^{\star} P<0.05,{ }^{\star \star} P<0.01,{ }^{\star \star \star} P<0.001$.) $\mathbf{g}$, ZIKV vRNA levels from brain of infected dams (2016 ZIKV $\mathrm{LD}: n=6$ and 14 days post-infection (dpi) $n=14 ; 2016 Z_{\text {IKVV }}: 3$ dpi $n=13$, and 6 and 14 dpi $n=10$; presented as copies per $\mu$ lafter normalization to a standard curve of $Z I K V$ RNA, values are shown as means). $\mathbf{h}$, Detection of viral RNA by fluorescence in situ hybridization (FISH) in the brain of ZIKV-infected dams (representative pictures are shown; PBS: $n=5 ; 2016 \mathrm{ZIKV}_{\mathrm{LD}}: n=6$ ). Positive signal (yellow) present in the brainstem (arrows) and in the cerebellum (arrows) within the white (WM) and grey matter (GM). As a negative control, a non-ZIKV-specific probe was used (control). Scale bars, $100 \mu$ m. i, Human neural progenitor cells (NPCs) were infected with 2016 ZIKV at different multiplicities of infection (MOIs). At the indicated time points, virus titres were determined by plaque assay ( $n=3$ ). Total numbers are shown as $n$ and represent biologically independent animal samples or experiments as indicated. hpi, hours post-infection; PFU, plaque-forming unit. 

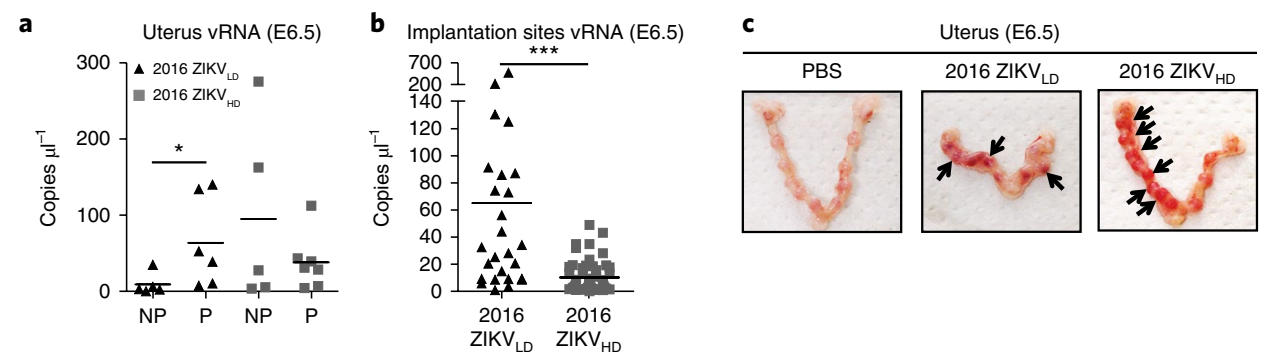

d

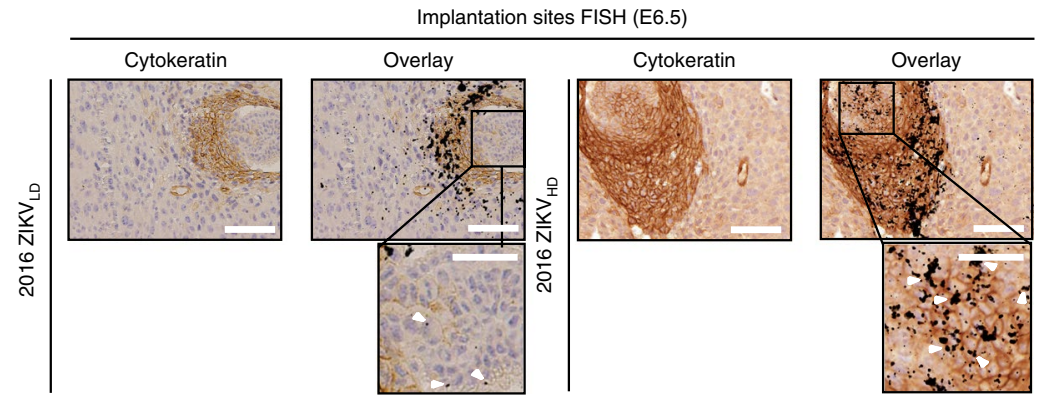

e
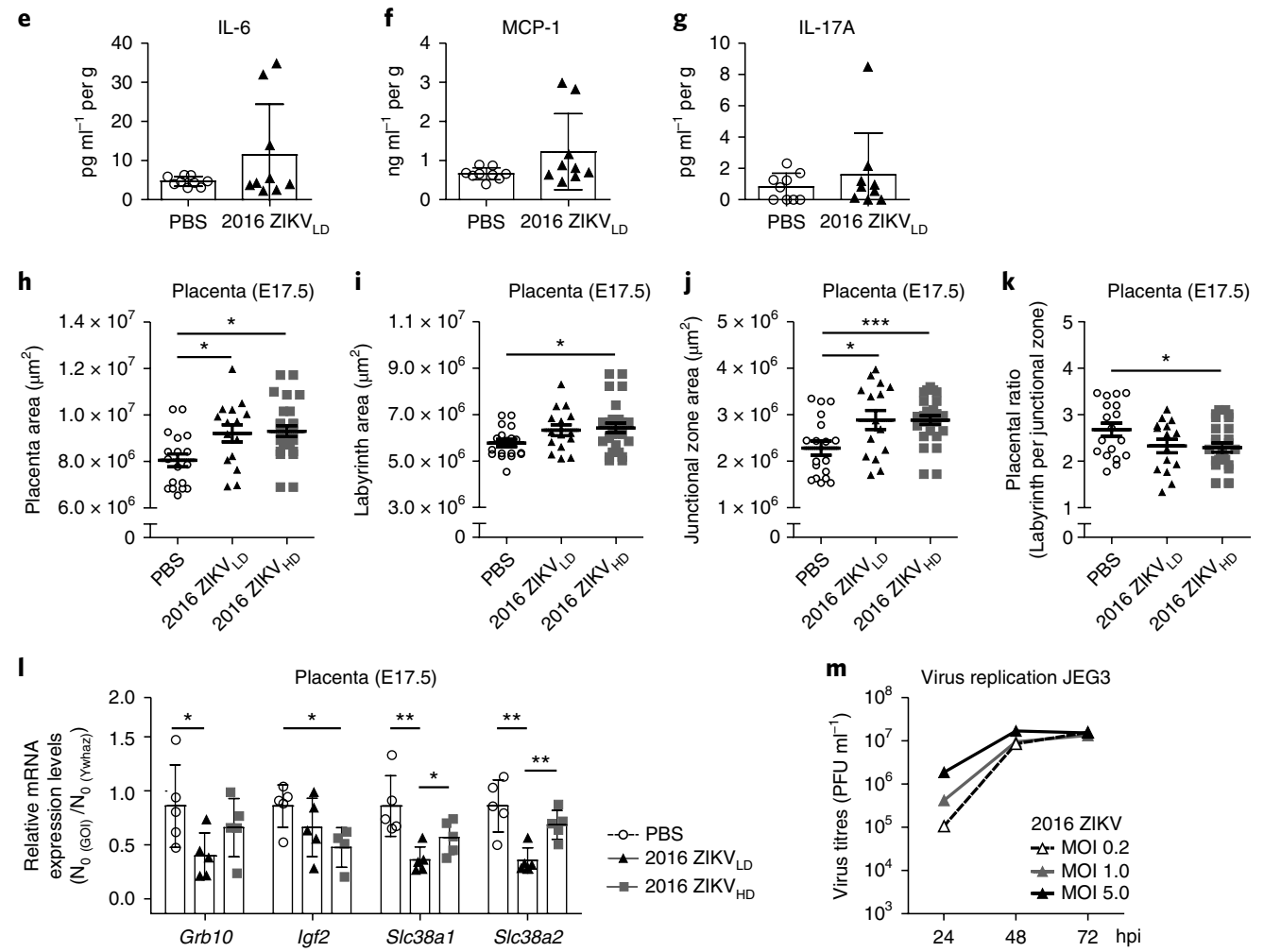

Fig. 2 | Vertical ZIKV transmission. a, ZIKV RNA levels from the uteri of non-pregnant (NP) and pregnant $(P)$ infected mice $\left(2016 \mathrm{ZIKV} \mathrm{LD}_{\mathrm{LD}}: \mathrm{NP} n=5\right.$ and $P n=6 ; 2016 Z_{I K V_{H D}}: N P n=5$ and $\left.P n=7\right)$. b, Viral burden from single implantation sites of three or seven ZIKV-positive uteri $\left(2016 Z I K V_{L D}: n=26 ; 2016\right.$ $Z \mathrm{ZIKV}_{\mathrm{HD}}: n=60$; only positive samples are depicted in the graph). (a,b, Presented as copies per $\mu$ after normalization to a standard curve of ZIKV RNA; values are shown as means, two-way ANOVA, Bonferroni post hoc tests, ${ }^{\star} P<0.05$, ${ }^{\star \star \star}{ }^{\circ} P<0.001$.) $\mathbf{c}$, Uteri from ZIKV-infected dams (the arrows indicate severe red discoloration; PBS: $\left.n=5 ; 2016 \mathrm{ZIKV}_{\mathrm{LD}}: n=6 ; 2016 \mathrm{ZIKV}_{\mathrm{HD}}: n=5\right)$. d, Cytokeratin staining and detection of viral RNA by applying ZIKV-specific probes in the uteri of ZIKV-infected dams (representative pictures are shown; PBS: $n=50 ; 2016 \mathrm{ZIKV} \mathrm{LD}: n=55 ; 2016 \mathrm{ZIKV}$ HD: $n=51$ ). Positive signal for ZIKV RNA (black) present in the embryo is indicated by the arrow heads. Scale bars, $100 \mu$ m; inset, $50 \mu \mathrm{m}$. e-f, Cytokines IL-6 (e), MCP-1 (f; also known as CCL2) and IL-17A ( $\mathbf{g}$ ) detected in maternal placenta ( $n=9$; values are shown as means, error bars as s.d.). h-k, Placenta area (h), labyrinth area (i), junctional zone area $(\mathbf{j})$ and placental ratio by labyrinth per junctional zone area (k) obtained by histomorphometric analyses of Masson-stained placental tissue sections (PBS: $n=18 ; 2016 \mathrm{ZIKV}_{\mathrm{LD}}: n=15 ; 2016 \mathrm{ZIKV}_{\mathrm{HD}}: n=27$; values are shown as means, error bars as s.e.m.). I, Relative mRNA expression of nutrient supply genes of placenta from ZIKV-infected dams. The relative expression of PBS was set to 1 for each gene after normalization to $Y w h a z(n=5$; values are shown as means, error bars as s.e.m.). (h-I, Two-tailed Student's $t$-test, ${ }^{\star} P<0.05,{ }^{\star \star} P<0.01,{ }^{\star \star \star} P<0.001$.) $\mathbf{m}$, Human placenta epithelial cells (JEG-3) were infected with 2016 ZIKV at different MOls. At the indicated time points, virus titres were determined by plaque assay $(n=2)$. Total numbers are shown as $n$ and represent biologically independent animal samples or experiments as indicated. 
at early gestation. The presence of elevated IgM antibody levels in adult offspring was further confirmed by immunofluorescence in selected brains (Supplementary Fig. 3a). Further, we analysed whether maternal ZIKV infection affects placental cytokine/chemokine response that could affect fetal outcome. No significant differences in IL-6, MCP-1 and IL-17A expression levels were detected at E17.5 in maternal placenta tissues (Fig. 2e-g) unlike in maternal sera. The placental labyrinth and the junctional zone, where nutrient exchange occurs and hormones are produced, were significantly increased in LD and HD ZIKV-infected dams (Fig. 2h-k). The ratio of labyrinth over junctional zone, which indicates placental function, was significantly reduced in HD ZIKV-infected mothers. This indicates a placental insufficiency due to reduced exchange in the labyrinth ${ }^{21,22}$, opposed by an increased hormone production in the enlarged junction zone, possibly as an adaptive mechanism to maintain the pregnancy. An increase in placenta size correlates with adverse pregnancy outcomes, such as low birth weight in humans, probably due to insufficient transfer of nutrients from mother to fetus ${ }^{21,22}$. Transcription levels of growth factor receptor bound protein 10 (Grb10), insulin like growth factor 2 (Igf2) and two amino acid transporter genes Slc38a1 (solute carrier family 38 member 1) and Slc38a2 (solute carrier family 38 member 2) were significantly reduced in LD and HD ZIKV-infected maternal placenta tissues (Fig. 21). Furthermore, the clinical 2016 ZKV strain efficiently replicated in a human trophoblast cell line (Fig. $2 \mathrm{~m}$ ) consistent with our findings in mice. Thus, our murine model supports ZIKV transmission from mother to fetus probably via the cross-placental route as reported before in immunodeficient mice ${ }^{10}$ and humans ${ }^{6}$.

Male offspring are less likely to survive maternal 2016 ZIKV infection compared to their female littermates. Litter sizes in LD and HD ZIKV-infected mothers were lower compared to uninfected controls (Fig. 3a) with a high fetal loss rate in the HD ZIKV group (Fig. 3b). Early miscarriages were also reported in ZIKVinfected pregnant women ${ }^{5}$. Of various fetal and maternal tissues tested at E17.5 (fetal head, yolk sac, umbilical cord and placenta), only the yolk sac embedding the HD ZIKV fetus was virus-positive (Fig. 3c). Small fetuses were particularly frequent in dams with large litter sizes, while those with smaller litters had more fetal demise (Supplementary Fig. 3b). Fetuses that survived ZIKV infection presented a significantly reduced birth weight in both LD- and particularly HD-infected groups compared to those born to uninfected mothers (Fig. 3d). Fetuses born to HD ZIKV-infected mothers were further opposed in size to fetuses born to LD ZIKV-infected mothers (Fig. 3e,f; Supplementary Fig. 3c). However, reduced HD ZIKV fetal size was not associated with any developmental delays according to the Theiler stages (Supplementary Fig. 3d) representing offspring being small for gestational age. Most significantly, male offspring were less likely to survive in utero ZIKV infection compared to their female littermates. With increasing maternal ZIKV infection dose, survival rates of male offspring reduced while female survival significantly increased (Fig. $3 \mathrm{~g}, \mathrm{~h}$ ). To control that the observed effects on reproductive outcome are specific to 2016 ZIKV infection, we analysed fetal outcome in DENV2- and 1947 ZIKVinfected mothers. There, no differences in litter size, fetal loss, fetal weight or sex-specific survival rates were observed (Supplementary Fig. $3 \mathrm{e}-\mathrm{j}$ ) further highlighting that the detected sex-specific survival on maternal infection is a feature of the 2016 ZIKV outbreak strain. In utero conditions may affect reproductive outcome particularly if they occur during early fetal development ${ }^{23}$. This could be confirmed in our murine model, where 2016 LD ZIKV infection of pregnant mice during late gestation (E12.5) did not result in any differences in maternal weight gain, litter size, fetal loss or fetal weight (Supplementary Fig. 4). These findings further support the concept that there is a critical window (early gestation) during pregnancy, where maternal infection results in adverse reproductive outcome. Next, we addressed the question of whether there might be a specific link between abnormal maternal serum TST levels and sex specificity in the offspring. Fetuses born to ZIKV-infected mothers displayed elevated serum TST levels at E17.5 depending on the maternal ZIKV infection dose (Fig. 3i). In adult offspring, no significant differences in serum TST levels were observed in females born to LD ZIKV-infected mothers compared to females born to PBS-treated dams (Fig. 3j). Remarkably, adult males born to LD ZIKV mothers clustered in two groups: those with increased (up to 3 times; referred as TST high) and those with very high (up to 50 times; referred as TST storm) TST levels unlike males born to uninfected mothers. In control experiments, elevated TST levels could not be observed in adult offspring born to DENV2- or 1947 ZIKVinfected mothers (Supplementary Fig. 3k,l) further highlighting the direct impact of the epidemic caused by the 2016 ZIKV infection on reproductive outcome and sex.

\section{Maternal TST treatment causes pregnancy termination but does} not affect the sex of the offspring. We then addressed the question of whether there is a causal relationship between increased maternal TST levels and reproductive outcome. Single TST injection or continuous release of TST at low levels $\left(0.05 \mathrm{mg} \mathrm{ml}^{-1}\right)$ did not affect maternal weight gain, reproductive outcome or the sex of the offspring (Supplementary Fig. 5, Fig. 3k-n). Conversely, very high levels of continuous TST release $\left(5 \mathrm{mg} \mathrm{ml}^{-1}\right)$ resulted in early pregnancy termination. Treatment of pregnant mice with a mid-range dose of $0.5 \mathrm{mg} \mathrm{ml}^{-1}$ resulted in two groups. Two of seven treated mice showed a delay in gestational weight gain and all fetuses were stillborn. Five of seven treated mice did not show differences in gestational weight, reproductive outcome or the sex of the offspring (Fig. 3k-n). These findings suggest that elevated TST levels during early gestation may affect pregnancy maintenance and reproductive outcome but do not influence sex differences in the surviving offspring. These findings are in line with previous reports that prenatal TST exposure in large mammals may result in intrauterine growth restriction in offspring without any sex bias ${ }^{24}$. Even though human studies are limited, there is growing evidence that adverse conditions during gestation may lead to dimorphic effects ${ }^{23}$. Herein, particularly malnutrition is a known parameter to affect sex ratios towards female offspring ${ }^{25}$. Since maternal ZIKV infection resulted in placental insufficiency in our murine model, reduced nutrient transfer from mother to fetus might be a potential contributor to reduced male but increased female in utero survival.

TST levels are elevated in ZIKV RNA-positive pregnant women and their newborns. We sought evidence whether our findings in the murine pregnancy model would be reflected in humans. Therefore, we analysed patient sera obtained from a mother-child cohort. ZIKV RNA-positive pregnant women showed elevated TST levels compared to ZIKV RNA-negative pregnant women. The elevated TST levels of the ZIKV RNA-positive pregnant women were also reflected in their newborns, unlike those children born to ZIKV RNA-negative mothers (Fig. 4). These data clearly reflect our findings in the murine model and suggest that elevated TST levels in children born to ZIKV RNA-positive mothers require further attention.

\section{Male offspring present more severe neuropathological altera-} tions in the hippocampus compared to their female littermates. TST plays a key role in fetal neurodevelopment ${ }^{18,26}$. Therefore, we addressed the question of whether murine offspring with elevated TST levels born to ZIKV-infected mothers may suffer from silent neuropathological disorders. None of the HD or LD fetuses presented ZIKV RNA in their brains on the day of birth (E17.5) (Supplementary Fig. 6a). However, fetal brains presented IgM antibodies against ZIKV, suggesting that active virus replication 

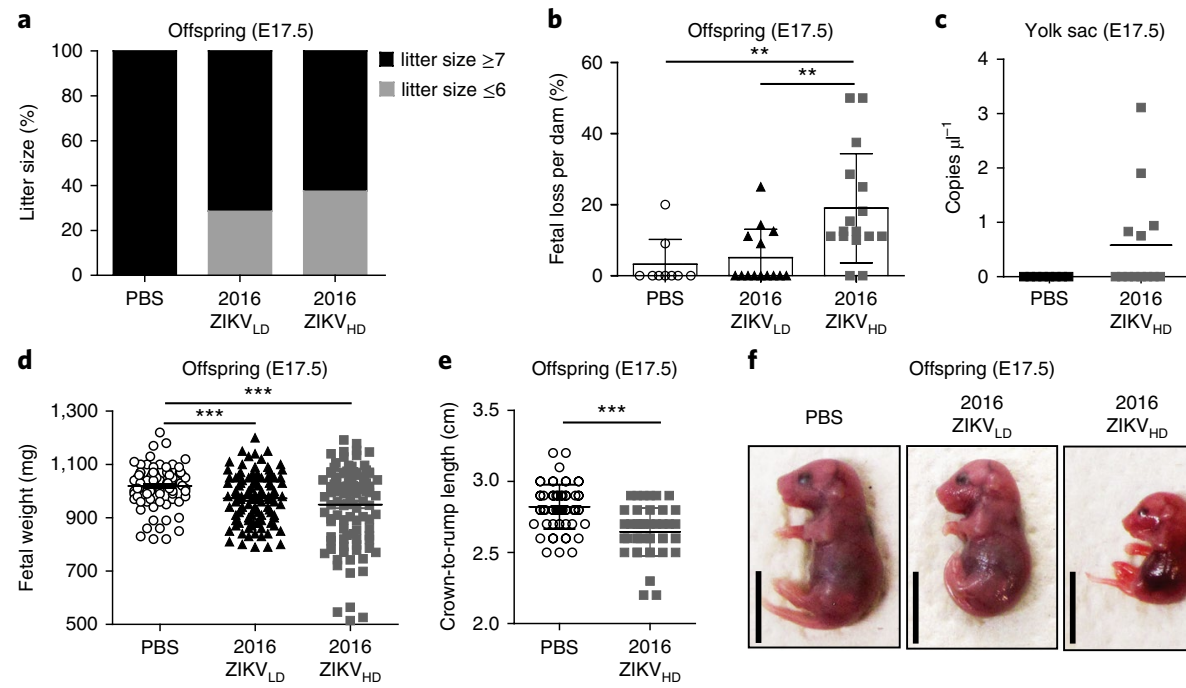

f
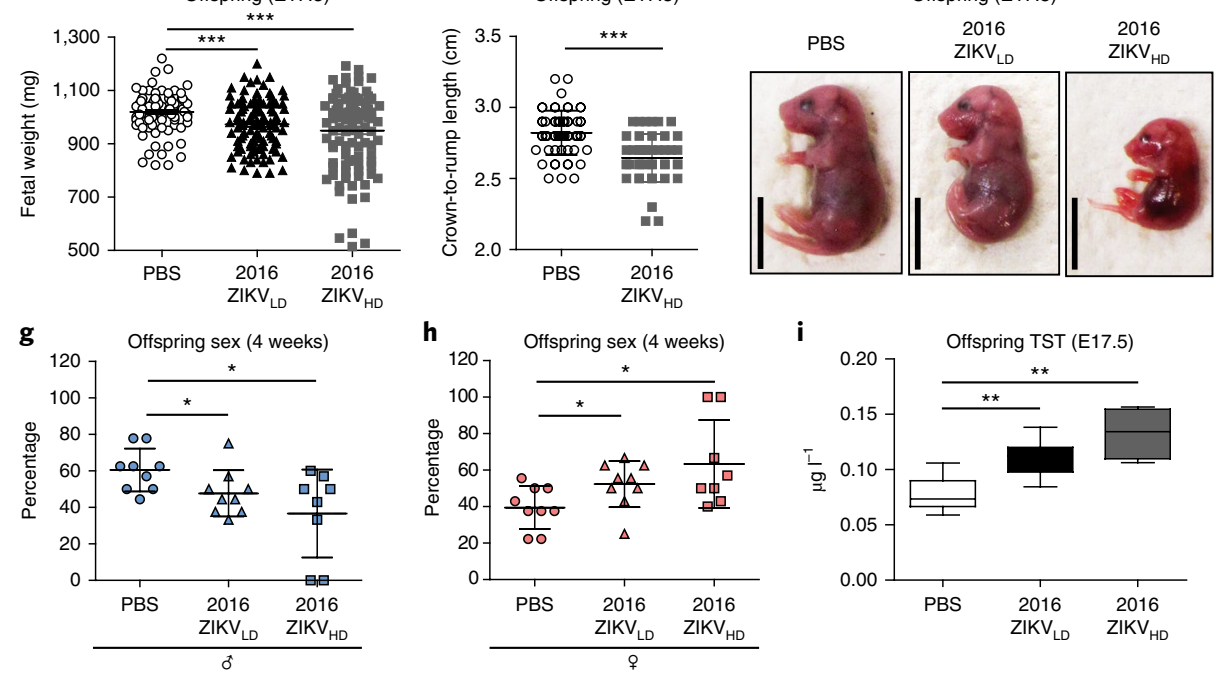

j

k
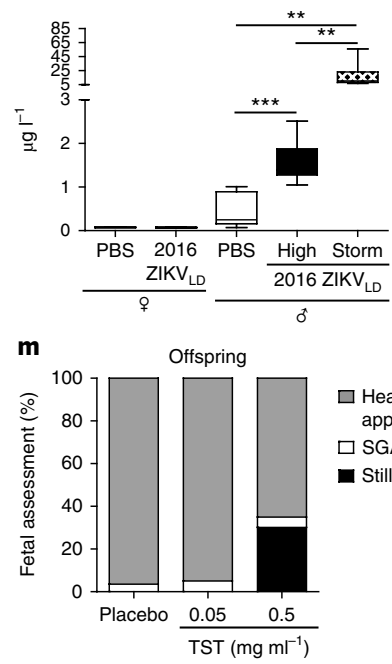

h
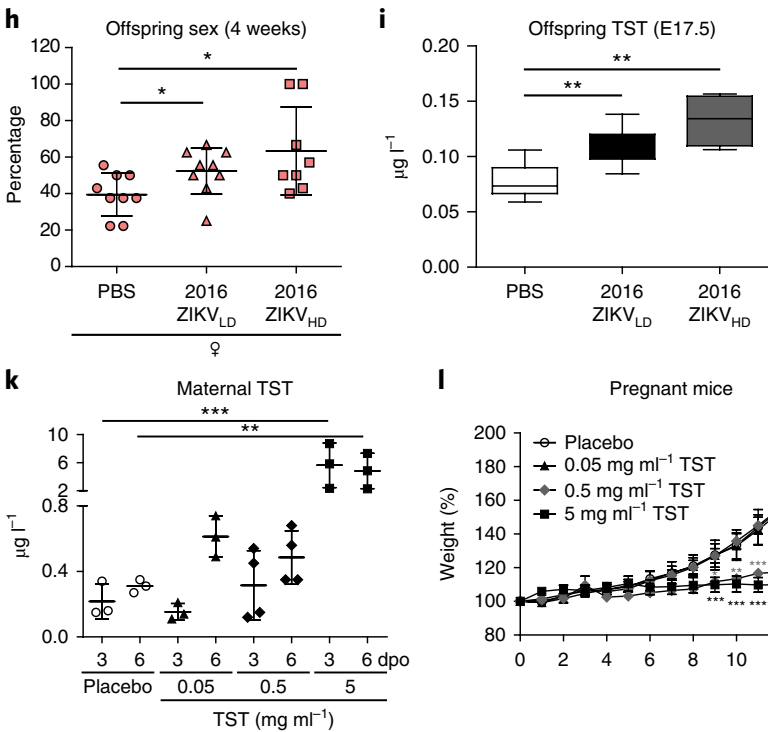

I
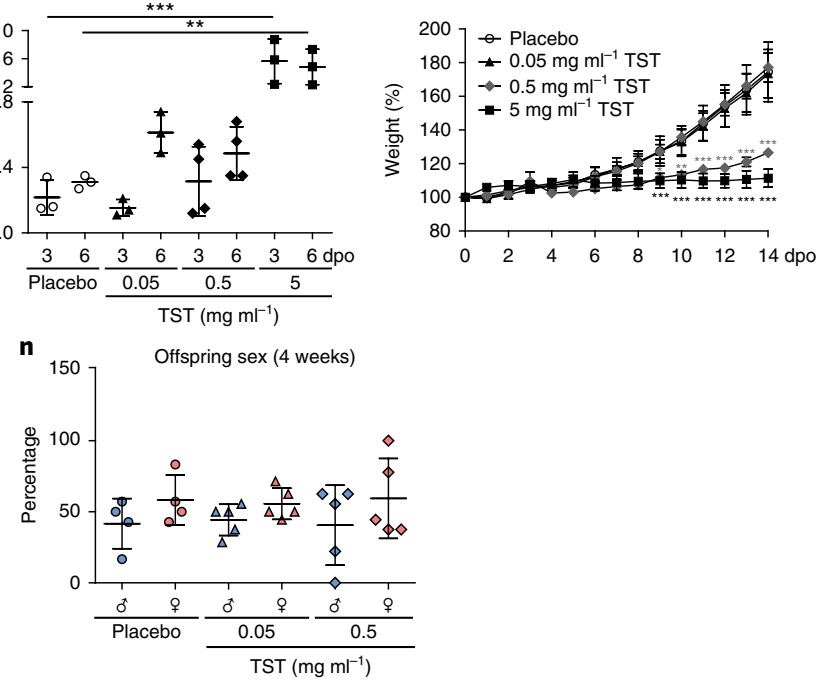

Fig. 3 Reproductive outcome. a, Litter size of ZIKV-infected dams (PBS: $n=10 ; 2016 \mathrm{ZIKV}_{\mathrm{LD}}: n=14 ; 2016 \mathrm{ZIKV}_{\mathrm{HD}}: n=16$; grouped in large ( $\geq 7$ fetuses) or small ( $\leq 6$ fetuses) litters). $\mathbf{b}$, Frequency of fetal loss shown as the total number of dead fetuses divided by the total number of healthy and dead fetuses per dam (PBS: $n=10 ; 2016 Z_{\text {ZIKV }}: n=14 ; 2016 Z_{L I K V}$ : $n=16$ ). c, ZIKV RNA levels from the yolk sac of infected dams (values are shown as means; PBS: $n=8 ; 2016 Z_{Z} I K V_{H D}: n=13$; presented as copies per $\mu$ after normalization to a standard curve of ZIKV RNA). d, Fetal weight of offspring from ZIKV-infected dams (PBS: $n=94 ; 2016 Z_{\text {ZIKV }}: n=109 ; 2016 Z_{\mathrm{LIKV}}$ : $n=108$ ). e, Crown-to-rump length of fetuses (PBS: $n=59 ; 2016$ ZIKV $\mathrm{HD}_{\mathrm{HD}}: n=44$ ). f, Offspring from ZIKV-infected dams. Scale bars, $10 \mathrm{~mm}$. $\mathbf{g}, \mathbf{h}$, Sex distribution of males $(\mathbf{g})$ and females $(\mathbf{h})$ in litters from ZIKV-infected dams (values are shown as means, error bars as s.d.; PBS: $n=9 ; 2016 \mathrm{ZIKV}_{\mathrm{LD}}: n=9 ; 2016 \mathrm{ZIKV}_{\mathrm{HD}}: n=8$ ). i,j, TST levels detected in serum offspring at E17.5 (i, values per litter; PBS: $n=6$; $2016 \mathrm{ZIKV}_{\mathrm{LD}}: n=5 ; 2016 \mathrm{ZIKV}_{\mathrm{HD}}: n=4$ ) and 4-8 weeks (j, values for individual animals; female, PBS: $n=10$ and 2016 ZIKV $: n=10 ;$ male, PBS: $n=9,2016$ ZIKV ${ }_{L D}$ : TST high $\left(1.05-2.51 \mu \mathrm{gl}^{-1}\right) n=9$ and 2016 ZIKV $_{\mathrm{LD}}$ : TST storm $\left.\left(6.77-56.15 \mu \mathrm{gl}^{-1}\right) n=13\right)(\mathbf{i}, \mathbf{j}$, Data presented as box plots, the whiskers indicating the minimum and maximum range of values.) $\mathbf{b}, \mathbf{d}, \mathbf{e}, \mathbf{g}-\mathbf{j}$, Values are shown as means, error bars as s.d., two-tailed Student's $t$-test, ${ }^{\star} P<0.05,{ }^{\star \star} P<0.01$, ${ }^{\star \star \star} P<0.001$. k. TST levels detected in maternal serum (placebo: $n=3 ; 0.05 \mathrm{mg} \mathrm{ml}^{-1} \mathrm{TST}: n=3 ; 0.5 \mathrm{mg} \mathrm{ml}^{-1} \mathrm{TST}: n=4 ; 5 \mathrm{mg} \mathrm{ml} \mathrm{l}^{-1} \mathrm{TST}: n=3$ ). I, Weight gain of pregnant mice implanted with TST pump $\left(0.05 \mathrm{mg} \mathrm{ml}^{-1} \mathrm{TST}: n=5 ; 0.5 \mathrm{mg} \mathrm{ml}^{-1} \mathrm{TST}: n=7 ; 5 \mathrm{mg} \mathrm{ml}^{-1} \mathrm{TST}: n=6\right)$ or placebo pump $(n=4)$. (k,l, Values are shown as means, error bars as s.d., two-way ANOVA, Bonferroni post hoc tests, $\left.{ }^{\star} P<0.05,{ }^{\star \star} P<0.01,{ }^{\star \star \star} P<0.001\right)$. $\mathbf{m}$, Fetal health assessment per litter of pump-implanted dams (placebo: $n=4 ; 0.05 \mathrm{mg} \mathrm{ml}^{-1} \mathrm{TST}: n=5 ; 0.5 \mathrm{mg} \mathrm{ml}^{-1} \mathrm{TST}: n=7$; grouped in healthy-appearing, small for gestational age (SGA) and stillborn). $\mathbf{n}$, Sex distribution in litters from pump-implanted dams (values are shown as means, error bars as s.d.; placebo: $n=4$; TST each: $n=5$ ). Total numbers are shown as $n$ and represent biologically independent animal samples as indicated. 


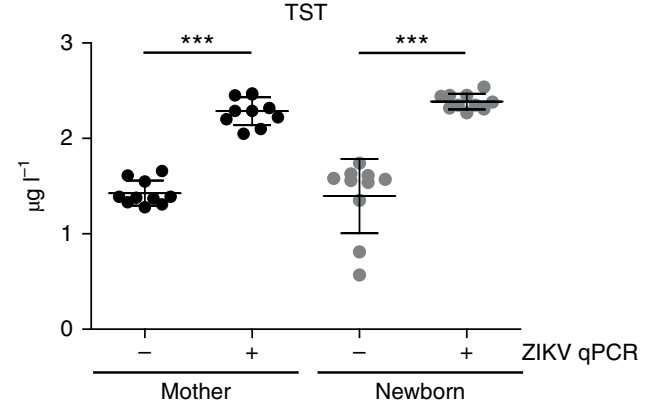

Fig. 4 | TST levels of pregnant women and newborns. TST levels were detected in sera obtained from ZIKV RNA-positive and ZIKV RNA-negative mothers during their last term of pregnancy as well as post-partum from their clinically inapparent newborns within the first month of life $(n=10$; values are shown as means, error bars as s.d., two-tailed Student's t-test, $\left.{ }^{\star \star \star} P<0.001\right)$. Total numbers are shown as $n$ and represent individual patients. qPCR, quantitative PCR.

was probably cleared to undetectable levels at birth. Supportively, no differences in brain MCP-1 or IL-17A levels were detected (Supplementary Fig. 6b,c) further indicating successful viral clearance. However, increased apoptotic cells in the brainstem could be detected compared to non-infected controls (Supplementary Fig. $6 \mathrm{~d})$. The same brain regions showed increased numbers of ionized calcium-binding adapter molecule 1 (Iba1)-positive cells indicative of microglia activation ${ }^{27}$ as a marker of inflammatory response of the brain probably representing the remains of previous active virus replication (Supplementary Fig. 6e). Microglial activation could no longer be detected in the brains or retina reported to support ZIKV replication ${ }^{28}$ in adult mice (Supplementary Fig. 7). The observed neuropathology in our murine model is consistent with the clinical case described, where the isolated 2016 ZIKV displayed abundant apoptosis and macrophage infiltrates in the fetal brain ${ }^{2}$. Brain inflammation with activated microglia as well as differential TST exposure in utero may affect sexual differentiation of brain structures and learning in adulthood, particularly in males ${ }^{18}$. Therefore, we analysed whether the detected alterations in these parameters may affect the adult offspring brain on maternal LD ZIKV infection, focusing on the hippocampus, which is known to support ZIKV replication and plays a key role in neurocognitive disorders ${ }^{29}$. In particular, we examined hippocampal CA1 neurons in adult male and female offspring. The complexity of apical or basal dendrites was similar in all offspring born to LD ZIKV-infected mothers (Supplementary Fig. 8 and 9). In distal dendritic branches from apical dendrites, both female and male LD ZIKV offspring presented increased spine densities (Fig. 5a-d). However, apical spine maturity (mushroomshaped neurons) was only affected in male but not female offspring. Male offspring had a significantly reduced number of mushroomshaped mature spines in apical dendrites unlike female offspring (Fig. 5e-h). Similarly, in basal dendrites, we could detect that neurons of male offspring showed increased spine density compared to neurons from PBS-treated controls (Fig. 5i,j). Conversely, the basal dendrites of female offspring presented reduced spine densities compared to their respective controls (Fig. 5k,1). However, both LD ZIKV male and female offspring presented significantly less mushroom-shaped spines in the analysed basal branches compared to their controls (Fig. $5 \mathrm{~m}-$ p). Thus, males with elevated TST levels in general (irrespective of its concentration since both TST high and TST storm males showed similar defects (Supplementary Fig. 10)) present a more severe brain defect with an increased number of immature spines in apical and basal dendrites in contrast to females, which do not have elevated TST levels and show more immature spines in basal neurons only (Supplementary Table 3).
Male offspring with high but not storm TST levels show alterations in behaviour and hippocampal learning deficiencies. The hippocampus has been implicated before in behavioural responses to stress and in the pathophysiology of mood and anxiety disorders ${ }^{30}$. Thus, we addressed the question of whether the differences detected in brain morphology between males and females might result in potential differences in learning and memory or may cause anxiety disorders in their adulthood. First, we analysed visual function by assessing spontaneous exploratory behaviour in the virtual drum of all LD ZIKV offspring to exclude that any potential alterations in behaviour studied later might be due to visual impairments in the offspring. However, visual acuity was not significantly affected in any of the LD ZIKV offspring assessed (females as well as TST high and TST storm males) (Supplementary Fig. 11a). In the novel environment of the open field arena, performance of male offspring born to LD ZIKV-infected mothers strongly depended on TST concentrations. TST high males showed most profound impairments in the exploration of the open field as indicated by distance moved and centre time (Fig. 6a,b). In contrast, TST storm males did not show any significant differences in behaviour as assessed in the open field (Fig. 6a,b). Female offspring born to LD ZIKV-infected mothers also showed a reduced exploration behaviour in the open field as indicated by a marginal but still significant reduced distance moved (Fig. 6a) and the time spent in the centre (Fig. 6b). No differences in behaviour were detected in LD ZIKV offspring in the Y-maze or in object-recognition tests (Supplementary Fig. 11b,c). These findings suggest that TST high males are at risk for potential anxiety disorders as assessed in the open field test compared to females. This is in line with previous reports of a negative correlation between time spent in the centre of the open field and neuropathological alterations in the brain caused by MIA $^{14}$ (Supplementary Table 3). However, male offspring with extremely high TST levels seem to be able to compensate for some behavioural alterations despite substantial brain defects, at least those functions assessed in this particular behavioural test. Next, we performed the Morris water maze (MWM) test to assess whether sex-specific differences in brain morphology might affect spatial (hippocampus-dependent) learning and memory ${ }^{31}$. All LD ZIKV offspring learned to find the hidden platform over the ten days of the experiment (Fig. $6 \mathrm{c}-\mathrm{d}$ ). However, inter-group differences were particularly observed in the search strategies described previously ${ }^{32}$ (Fig. 6e-g). First, most mice preferred either to swim along the wall of the pool (thigmotactic or wall-hugging swim ${ }^{32}$ ) or found the hidden platform by chance with occasional inner area searches of the pool $^{32}$. Later, as training progressed, mice started to search the entire pool surface area, first randomly, and later by selective scanning of the inner pool area with the escape platform. Finally, focal search of the target quadrant containing the platform, or a direct swim strategy towards it, suggested the development of spatial memory ${ }^{32}$. The majority of PBS male offspring changed their search strategy from random or systematic (non-spatial) to spatial over the ten days of testing, while LD ZIKV male offspring used non-spatial search strategies significantly more often, indicating a hippocampal deficit (Fig. 6f). This was particularly pronounced in TST high unlike TST storm males (Fig. 6f). No significant inter-group differences in search strategies were detected in female offspring, although a general tendency towards increased non-spatial search strategies was observed (Fig. 6g). It has been previously shown that mice can change their search strategy from spatial (hippocampus-dependent) to systematic non-spatial (striatum-dependent) after disruption of hippocampal function $^{33,34}$. These findings show that TST high males present a hippocampal deficit in learning strategies unlike females and TST storm males. Similar to the findings in the open field assessing mainly anxiety, hippocampal learning is different between females and TST high males. Consistently, TST storm males could compensate for deficiencies in learning strategy suggesting a dose-dependent function of TST (Supplementary Table 3). 

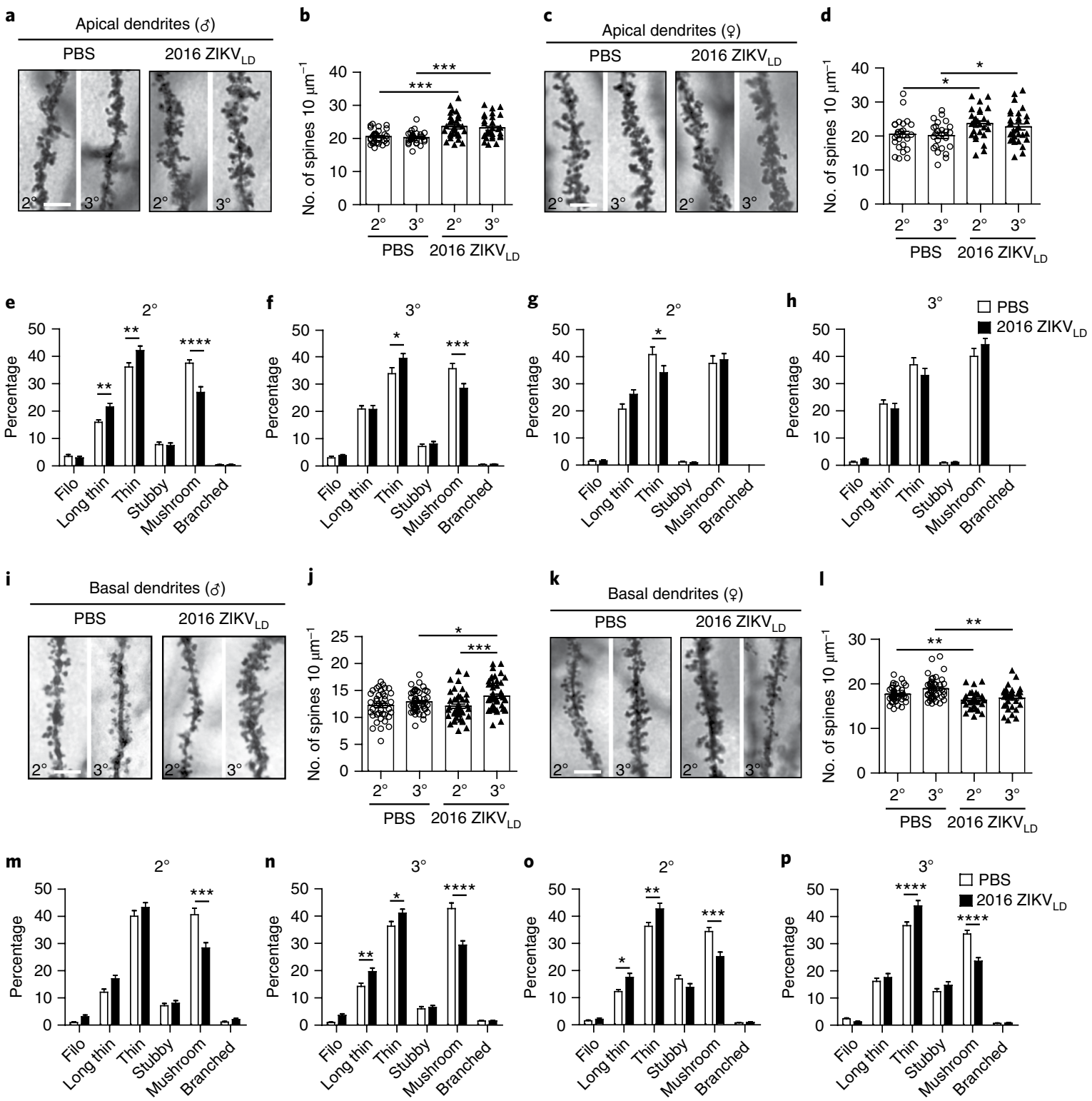

Fig. 5 | Apical and basal dendrites of adult male and female offspring. Spine density and distribution of spine types in apical (a,c) and basal (i,k) dendrites of CA1 pyramidal neurons in secondary and tertiary branches from male (a,i) and female (c,k) offspring of control and ZIKV-infected dams. $\mathbf{a}, \mathbf{c}$, Spine density in apical dendrites from male (a) and female (c) offspring. Scale bar, $5 \mu \mathrm{m}$. $\mathbf{b}, \mathbf{d}$, Spine density quantification of apical dendrites of $C A 1$ pyramidal neurons from male (b, PBS: secondary $\left(2^{\circ}\right) n=30$ and tertiary $\left(3^{\circ}\right) n=28 ; 2016 Z_{\text {ZIKV }}$ :D $n=30$ segments from 7 cells from 4 brains) and female (d, PBS: $n=25$ segments from 6 cells from 3 brains; $2016 Z_{\text {IIKV }}$ : $2^{\circ} n=28$ and $2^{\circ} n=30$ segments from 6 cells from 4 brains) offspring (values are shown as means, error bars as s.e.m., two-tailed Student's $t$-test, $\left.{ }^{\star} P<0.05,{ }^{\star \star \star} P<0.001\right)$. e-h, Distribution of spine types in apical dendrites of $2^{\circ}$ branches for male offspring (e, PBS: $n=30 ; 2016 \mathrm{ZIKV}_{\mathrm{LD}}: n=30$ dendritic segments) and female offspring (g, PBS: $n=25,2016$ ZIKV $\mathrm{LD}_{\mathrm{LD}}: n=26$ dendritic segments) and $3^{\circ}$ branches for male offspring (f, PBS: $n=28 ; 2016$ ZIKV $_{\mathrm{LD}}: n=30$ dendritic segments) and female offspring (h, PBS: $n=25 ; 2016$ ZIKV $\mathrm{LD}: n=26$ dendritic segments; values are shown as means, error bars as s.e.m., two-way ANOVA with Šidák's multiple comparison). i,k, Spine density in basal dendrites of CA1 pyramidal neurons from male (i) and female (k) offspring. Scale bar, $5 \mu \mathrm{m}$. j,l, Spine density quantification of basal dendrites of CA1 pyramidal neurons from male (j, PBS: $2^{\circ} n=40$ and $3^{\circ} n=50 ; 2016 \mathrm{ZIKV}_{\mathrm{LD}}: 2^{\circ} n=43$ and $3^{\circ} n=50$ segments from 8 cells from 3 brains) and female (I, PBS: $n=40$; 2016 ZIKV $\mathrm{LD}: n=30$ segments from 8 cells from 3 brains) offspring (values are shown as means, error bars as s.e.m., two-tailed Student's $t$-test, ${ }^{*} P<0.05$, $\left.{ }^{\star \star} P<0.01,{ }^{\star \star \star} P<0.001\right)$. m-p. Distribution of spine types in basal dendrites of $2^{\circ}$ branches for male offspring ( $\mathbf{m}, P B S: n=38 ; 2016 \mathrm{ZIKV}$ : $n=43$ dendritic segments) and female offspring (o, PBS: $n=40 ; 2016 \mathrm{ZIKV}_{\mathrm{LD}}: n=30$ dendritic segments) and $3^{\circ}$ branches for male offspring (n, PBS: $n=47 ; 2016$ ZIKV $n=49$ dendritic segments) and female offspring (p, PBS: $n=40 ; 2016 \mathrm{ZIKV}_{\mathrm{LD}}: n=30$ dendritic segments; values are shown as means, error bars as s.e.m., two-way ANOVA with Šidák's multiple comparison, $\left.{ }^{\star} P<0.05,{ }^{\star \star} P<0.01,{ }^{\star \star \star} P<0.001,{ }^{\star \star \star \star} P<0.0001\right)$. Total numbers are shown as $n$ and represent biologically independent samples as indicated.

\section{Discussion}

Generally elevated TST levels in male offspring correlate with increased brain damage as assessed by the presence of immature neurons in apical and basal dendrites compared to female offspring, where immature spines were present in basal neurons only. Very high TST concentrations in TST storm males did not result in more severe morphological alterations in the brain suggesting that an elevated TST level in general is sufficient for profound brain damage (Fig. 6h). Consistently, elevated TST levels in TST high males also correlated with impairments in behaviour (Fig. 6i) and learning 

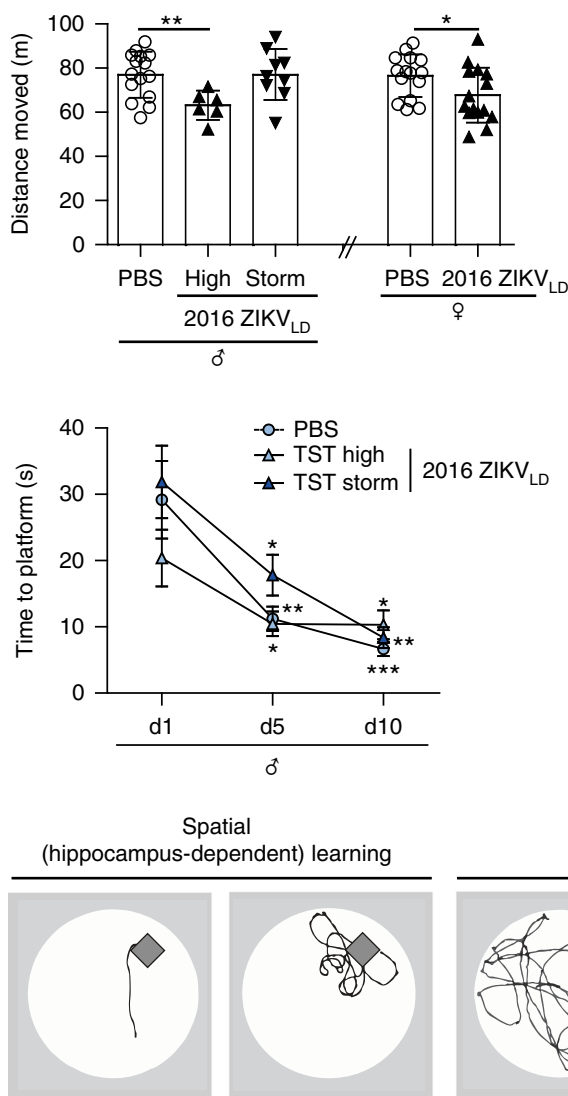

Spatial
Spatial

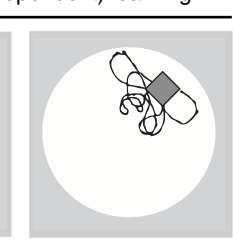

Focal
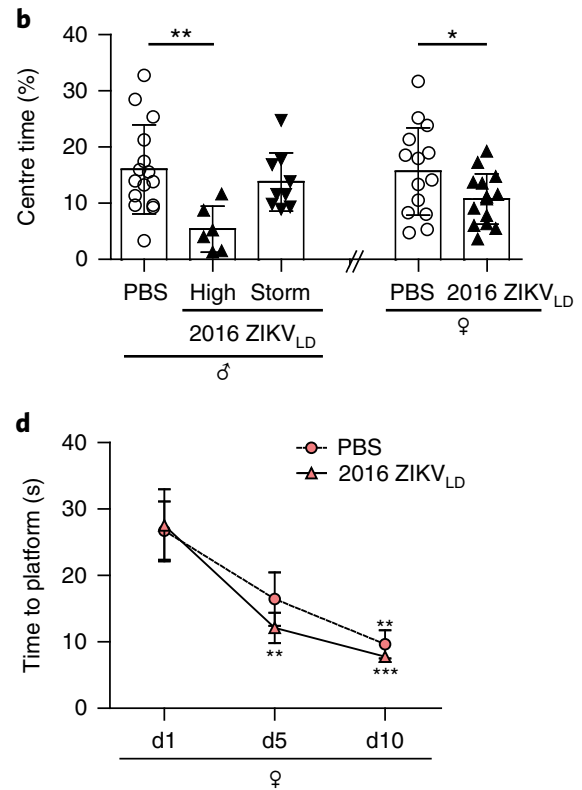

f

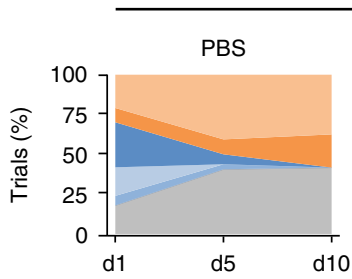

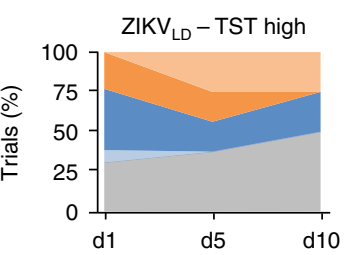

i

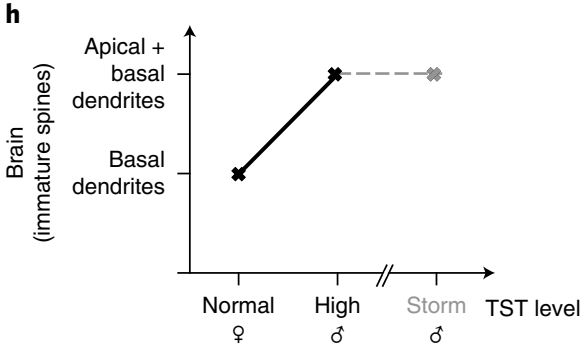

i

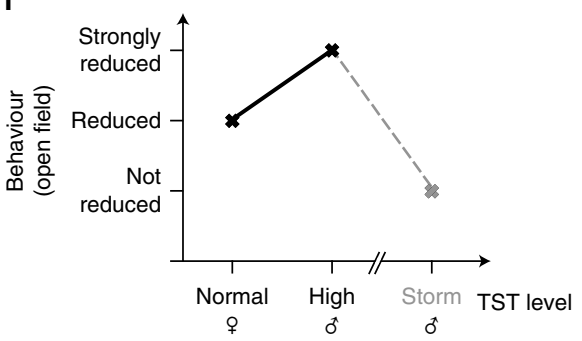

Non-spatial (striatum-dependent) learning

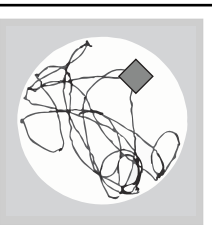

Scanning

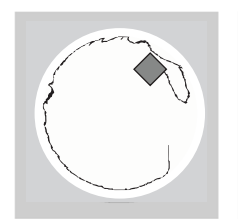

Thigmotaxis

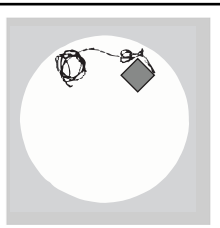

Circling

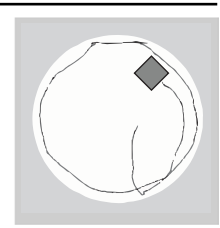

Chaining g

우
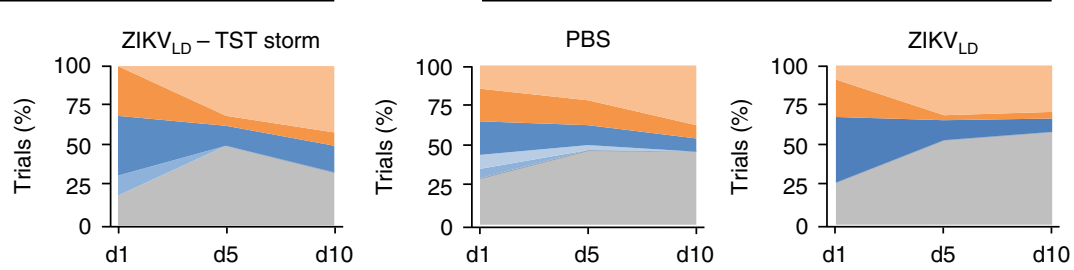

j

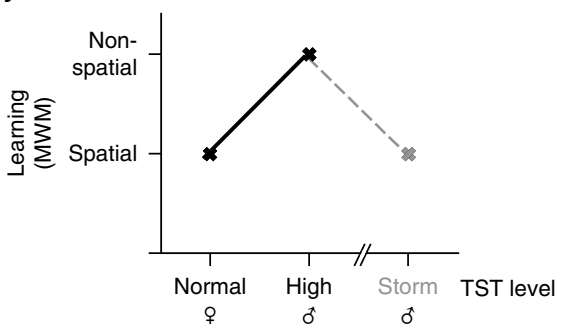

Fig. 6 | Assessment of spatial learning and memory of adult male and female offspring. a,b, Distance moved (a) and centre time in the first 5 min (b) in the open field test (values are shown as means, error bars as s.e.m., $n=15$ per group, except TST high ( $n=6$ ) and TST storm ( $n=9$ ); one-way ANOVA or unpaired two-tailed Student's $t$-test, ${ }^{\star} P<0.05,{ }^{\star \star} P<0.01$ versus PBS). $\mathbf{c}, \mathbf{d}$, Time to platform (escape latency) for male (c) and female (d) offspring from PBS and 2016 ZIKV $_{\text {LD }}$-infected dams in the MWM (values are shown as means, error bars as s.e.m., $n=8$ per group except TST high and TST storm $(n=4)$; ANOVA with Fisher's least significant difference test versus day $\left.1 ;{ }^{\star} P<0.05,{ }^{\star \star} P<0.01,{ }^{\star \star \star} P<0.001\right)$. Average swim velocity per trial ranged between 18 and $27 \mathrm{~cm} \mathrm{~s}^{-1}$ and was not significantly different between groups. e, Spatial (hippocampus-dependent) and non-spatial (striatum-dependent) search strategies used by individual mice in all groups. $\mathbf{f}, \mathbf{g}$, Search strategy use over the ten days of hidden platform testing. Search strategy use differed between male offspring of PBS and 2016 ZIKV $_{\mathrm{LD}}$-infected dams (f); the majority of PBS controls changed their search strategy from random (non-spatial)

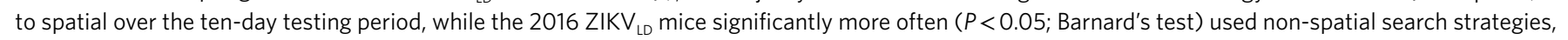
indicating hippocampal deficit. This was particularly pronounced in ZIKV LD mice with high TST levels (f). g, Search strategies used by female mice. h-j, Correlation of TST levels with brain morphology (immature spines in CA1 hippocampal neurons) (h), behaviour in the open field test (centre time) (i) or learning strategies (hippocampus dependency) in the MWM test (j) summarized from data shown under Figs. 3j, 5 and 6a-g and Supplementary Figs. 8-10. Total numbers are shown as $n$ and represent biologically independent animals. 
strategies (Fig. 6j) compared to females with normal TST levels. However, TST storm males did not show differences in behaviour and learning despite neuronal changes. As shown previously, brain structure is not always predictive of behaviour ${ }^{35}$. Moreover, effects of TST on behaviour were reported to be curvilinear rather than linear and particular sex-dependent (reviewed in Celec et al..$^{36}$ ). However, future studies are clearly required to assess the impact and the mode of action of TST concentrations on neurodevelopment and behaviour in later life.

In summary, our data support the concept that maternal ZIKV infection during early embryogenesis affects fetal development in utero. As a consequence, offspring that do not show any clinical manifestations at birth might suffer from neuronal abnormalities, as well as learning and memory deficiencies, in their adulthood. Since morphological alterations in adult offspring brain were not detectable by standard non-invasive imaging techniques (Supplementary Fig. 12), targeted monitoring becomes particularly important. Interestingly, silent neuropathologies were also reported recently in offspring born to ZIKV-infected pigtail macaques (Macaca nemestrina $)^{37}$ further supporting the findings in our immunocompetent allogenic murine pregnancy model. Taken together, our data suggest that a complex interplay of maternal and viral factors contribute to neurodevelopmental and memory impairments in offspring's later life (Supplementary Fig. 13). This study might provide a basis for future longitudinal clinical studies to identify children who might be at increased risk to develop subclinical neurocognitive impairments in later life to allow timely and adequate disease management.

\section{Methods}

Ethics statement. Animal experiments were performed in strict accordance with the guidelines of German animal protection law. All animal protocols were approved by the relevant German authority (Behörde für Gesundheit und Verbraucherschutz). Tests performed at the German Mouse Clinic were approved by the responsible authority of the district government of Upper Bavaria, Germany.

Viruses and cells. We used the 2016 outbreak strain, FB-GWUH-2016 ZIKV (herein referred to as $2016 \mathrm{ZIKV}$ ), belonging to the Asian lineage (Supplementary Fig. 1), which was isolated from a human fetal brain with substantial abnormalities at the University of Helsinki, Finland ${ }^{2}$. The mother, who did not have any known underlying diseases, was infected with ZIKV during her first trimester; the infection was detected after her return from Central America. Given the gravity of the disease burden, she decided to prematurely terminate the pregnancy at 21 weeks of gestation ${ }^{2}$. DENV2 strain 16681 was originally isolated from a patient from Thailand who suffered from dengue haemorrhagic fever ${ }^{38}$. The prototype ZIKV strain MR766 was isolated in 1947 from Rhesus monkeys in Uganda. It underwent serial cell passages but is still genetically more similar to the 2016 outbreak strain than the DENV2 isolate; therefore, it was used as a genetically related control group. All isolates were propagated and titrated on African green monkey kidney epithelial (Vero E6; ATCC CCL81) cells. Vero E6 and A549 cells (DSMZ Braunschweig) were grown in DMEM medium (SigmaAldrich), supplemented with 10\% fetal bovine serum (FBS; Biochrom), 1\% penicillin-streptomycin (Sigma-Aldrich), and 1\% L-glutamine (Sigma-Aldrich), and cultivated at $5 \% \mathrm{CO}_{2}, 96 \%$ relative humidity and $37^{\circ} \mathrm{C}$. Human placenta cells (JEG-3; provided by Udo Markert, University of Jena) were grown in Ham's F12 Nutrient Mixture (Thermo Fisher Scientific), supplemented with 10\% FBS, $1 \%$ penicillin-streptomycin and $1 \% \mathrm{~L}$-glutamine, and cultivated at $5 \% \mathrm{CO}_{2}$ at $37^{\circ} \mathrm{C}$. Human neural progenitor cells (hNPCs; provided by Priit Pruunsild and Hilmar Bading, Heidelberg University) were grown in DMEM-F12 medium containing $2 \mathrm{mM}$ GlutaMAX, 1\% N2 supplement, 2\% B27 supplement, $50 \mu \mathrm{M} \beta$ -mercaptoethanol, penicillin-streptomycin (1:200), $10 \mathrm{ng} \mathrm{ml}^{-1}$ epidermal growth factor (all from Thermo Fisher Scientific) and $10 \mathrm{ng} \mathrm{ml}^{-1}$ fibroblast growth factor 2 (PeproTech), and were cultivated at $5 \% \mathrm{CO}_{2}$ at $37^{\circ} \mathrm{C}$. All cell lines have been tested free of Mycoplasma contamination with the Venor GeM Classic Mycoplasma PCR Detection Kit (Minerva Biolabs).

ZIKV replication in cell culture. A549 cells were infected with ZIKV at a multiplicity of infection (MOI) of 0.2. JEG-3 and hNPCs cells were infected with ZIKV using MOIs of $0.2,1$ and 5 . Virus titration was performed at 24, 48 and $72 \mathrm{~h}$ post-infection on Vero E6 cells by plaque assay.

Animal experiments. Animal experiments were performed in strict accordance with the guidelines of German animal protection law. All animal protocols were approved by the relevant German authority (Behörde für Gesundheit und Verbraucherschutz; protocols 124/12 and 38/16). Female C57BL/6 and male
BALB/c mice (8-10 weeks old) were purchased from ENVIGO and were set up for semi-allogeneic mating. Mice were kept under standard housing conditions $\left(21 \pm 2{ }^{\circ} \mathrm{C}, 40-50 \%\right.$ humidity, food and water ad libitum) with a $12: 12$ light-dark cycle. Animals with an unclear pregnancy status were excluded from subsequent experiments. Non-pregnant and pregnant mice at embryonic (E) days E3.5 or E12.5 were anaesthetized with isoflurane and inoculated by the retrobulbar route with $1 \times 10^{5} 50 \%$ cell culture infectious dose $\left(\right.$ TCID $_{50}$ ) for LD ZIKV or $2.5 \times 10^{7}$ $\mathrm{TCID}_{50}$ for HD ZIKV in $100 \mu \mathrm{l} 1 \times \mathrm{PBS}$. The range of ZIKV infectious dose used was chosen to reflect flavivirus infectious particles detected during a mosquito bite $^{39}$. Control groups received $1 \times$ PBS. Full-term vital fetuses were counted to assess reproductive outcome on E17.5 gestation, corresponding to 1 day before birth, to avoid infected mothers killing their sick offspring directly after birth, which would limit the assessment of the reproductive outcome. For the TST treatment experiment, an ALZet Model 1002 micro-osmotic pump (Charles River) that releases TST or, as a control, a placebo was implanted subcutaneously during E3.5 gestation under isoflurane narcosis. Weight loss/gain was monitored for 14 or 5 days post-infection and blood samples were collected on days 1,3 and 6 postinfection. Blood was centrifuged for $10 \mathrm{~min}$ at $2,000 \mathrm{~g}$ and $4^{\circ} \mathrm{C}$, and the respective sera were stored at $-80^{\circ} \mathrm{C}$. On days 3,6 and 14 post-infection, at least 5 animals per group were sacrificed and their organs (head, spleen and uterus or yolk sac) as well as placenta, umbilical cord and fetuses were removed and stored in $4 \%$ formalin or in RNAlater RNA Stabilization Reagent (QIAGEN). Mother and fetal heads, as well as uteri and placentas, were fixed in $4 \%$ formalin and embedded in paraffin for further immunohistochemical and pathological analysis, as well as determination of viral burden. Formalin-fixed uteri were processed for sectioning, as described previously ${ }^{40}$. Uteri, yolk sacs, placentas and umbilical cords were kept in RNAlater RNA Stabilization Reagent for the determination of virus titres. Eyes from eight-week-old offspring from ZIKV-infected and PBS control dams were fixed in $4 \%$ paraformaldehyde for further immunohistochemical analysis.

Measurement of cytokine/chemokine and hormone levels. Maternal cytokine and chemokine levels in serum were measured using a custom-made Bio-Plex Pro Mouse Cytokine I 4-plex assay (Bio-Rad), analysing IL-6 and MCP-1 levels according to the manufacturer's instructions in a Bio-Plex 200 System with high-throughput fluidics (HTF; Bio-Rad) or for IL-17A by enzyme-linked immunosorbent assay (ELISA; EMELCA Bioscience). Cytokine levels in the placenta and fetal brain were measured using a Bio-Plex Pro Mouse Cytokine kit (Bio-Rad), analysing IL-6, IL-17A und MCP-1 levels according to the manufacturer's instructions in a Bio-Plex 200 System with HTF (Bio-Rad). Progesterone (Cayman Chemical) and corticosterone (ARBOR ASSAYS) levels in maternal serum were evaluated by ELISA following the manufacturer's instructions. For progesterone analysis, serum was diluted 1:100 or 1:200 and measured after $70 \mathrm{~min}$ of substrate incubation. Serum levels of oestradiol (Calbiotech) were also determined by ELISA according to the manufacturer's instructions, except for a $50 \%$ reduced incubation time during enzyme conjugate incubation, and a 1:2 dilution of samples using standard 0 as diluent. All ELISAs were measured on a Saphire2 ELISA microplate reader (Tecan) and evaluated using a four parameter logistic regression (MyAssays). To determine serum TST levels, a chemiluminescence immunoassay (ADVIA Centaur Testosterone II assay; Siemens Healthcare Diagnostics) was employed. Measurement was performed with the ADVIA Centaur XP (Siemens Healthcare Diagnostics).

Determination of viral titres. Viral RNA was isolated from serum or tissue using the QIAamp Viral RNA Mini Kit (QIAGEN) according to the manufacturer's instructions. ZIKV RNA levels were determined by quantitative reverse transcription real-time PCR (qRT-PCR) using the RealStar Zika Virus RT-PCR Kit 1.0 (altona Diagnostics). Recommendations from the manufacturer for sample preparation were incorporated into the RNA isolation procedure. These included the use of an internal control, which served both as a control for the sample preparation and as the RT-PCR inhibition control, as well as an extended dry spin step for $10 \mathrm{~min}$ at $17,000 \mathrm{~g}$ and room temperature. Approximately $50 \mathrm{mg}$ of RNAlater-fixed organs were homogenized with eight sterile, stainless steel beads (Ø $2 \mathrm{~mm}$; Retsch) in $160 \mu \mathrm{l}$ of diethyl pyrocarbonate-treated $1 \times \mathrm{PBS}$ at $30 \mathrm{~Hz}$ and $4^{\circ} \mathrm{C}$ for $6 \mathrm{~min}$ in the mixer mill MM400 (Retsch). Supernatants were then used for

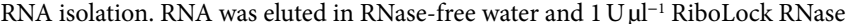
Inhibitor (Thermo Fisher Scientific) was added. Total RNA from formalin-fixed, paraffin-embedded (FFPE) mother or fetal heads was isolated using the RNeasy FFPE Kit (QIAGEN). Samples were deparaffinised using xylene. RNase-free DNAse Set (QIAGEN) was used for on-column DNase I treatment according to the manufacturer's instructions.

Detection of DENV2 non-structural protein 1. The DENV2 non-structural protein 1 (NS1) protein in mouse sera was detected by ELISA according to the manufacturer's instructions (EUROIMMUN AG). Measurements were performed at a $450 \mathrm{~nm}$ absorbance and a reference wavelength of $630 \mathrm{~nm} 20 \mathrm{~min}$ after the stop solution was added on a Saphire2 ELISA microplate reader.

Virus IgG and IgM assay. An immunofluorescence assay was performed in ZIKV-infected Vero E6 cells as described previously ${ }^{41}$. In brief, infected Vero E6 
cells were incubated with serial serum dilutions (1:20 and 1:160), washed with $1 \times$ PBS and stained with conjugated fluorescein isothiocyanate goat anti-mouse IgG antibody (Jackson ImmunoResearch). IgG titres of 1:20 or more were considered positive. For IgM detection, a commercial anti-ZIKV indirect immunofluorescence test (EUROIMMUN AG) was used according to the manufacturer's instructions. Serum samples were treated with the EzWay mouse IgG Depletion Solution (KOMABIOTECH) to pre-absorb IgG and rheumatic factors. Samples were diluted $1: 10$ and 1:20. Samples were evaluated by fluorescence microscopy with a 20 -fold magnification (Leica DMI6000), by comparing the fluorescence signals of ZIKVinfected versus non-infected cells.

Fluorescence in situ hybridization. Maternal FFPE tissue was cut at a $2-5 \mu$ $\mathrm{m}$ thickness and mounted on glass slides. For fluorescence in situ hybridization (FISH), an RNA probe was generated (VF1-19981-06, 6000098-06; Affymetrix) and used with the ViewRNA ISH Tissue Assay Kit (Thermo Fisher Scientific) as well as the ViewRNA Chromogenic Signal Amplification Kit (Thermo Fisher Scientific) according to the manufacturer's instructions. For RNA unmasking, a pretreatment buffer was used for $10 \mathrm{~min}$ at $85-90^{\circ} \mathrm{C}$, Protease QF (Affymetrix) was incubated for $20 \mathrm{~min}$, and $2.2 \mathrm{M}$ hydrogen chloride and $300 \mathrm{mM}$ potassium chloride were applied for $15 \mathrm{~min}$ as described previously ${ }^{10}$. Phase contrast and Cy3 fluorescence images were taken using a colour video camera (DP72, 12.8 megapixel CCD; Olympus) and an IX50 microscope (Olympus) with the cellF software (version 3.3; Olympus). The fluorescence signal was shown either in red, yellow or black in the respective figures to allow better visualization depending on the figure background using ImageJ (Fiji).

Histomorphological analyses of placental tissue. Paraffin-embedded placental tissue was cut into $6 \mu \mathrm{m}$ histological sections at the mid-sagittal plane using a microtome (Leica SM2010 R). Slides were allowed to dry and incubated overnight at $37^{\circ} \mathrm{C}$ before storing. Masson-Goldner trichrome staining kit (VWR) was used to visualize the morphologically different areas of placental tissue. After rinsing in distilled water, tissue sections were dehydrated twice in ethanol $70 \%$. Weigert's iron haematoxylin (Waldeck GmbH\&Co. KG) was prepared following the manufacturer's instruction and applied to the tissue sections. Slides were rinsed in tap water followed by $1 \%$ acetic acid in distilled water. Azophloxine staining solution (Goldner 1) and phosphotungstic acid-Orange G (Goldner 2) were subsequently added, followed by washing steps and counterstaining. The slides were mounted using Eukitt mounting media (O. Kindler ORSAtec $\mathrm{GmbH}$ ) and cover slips and examined using a ZEISS Axioscope light microscope. Photo documentation was performed with a digital image analysis system (KS400 software; ZEISS).

Determination of nutrient supply genes in the placenta. Total RNA isolation from placentas was conducted using the innuPREP RNA Mini Kit 2.0 (Analytik Jena) according to the manufacturer's instructions. Tissue homogenization of 30-40 mg of RNAlater-fixed placentas was performed in $450 \mu \mathrm{l}$ lysis buffer $\mathrm{RL}$ with 10 sterile, stainless steel beads ( $\varnothing 2 \mathrm{~mm}$; Retsch) at $30 \mathrm{~Hz}$ and $4{ }^{\circ} \mathrm{C}$ for $6 \mathrm{~min}$ in the mixer mill MM400. Supernatants were then used for RNA isolation. RNase-free DNase Set was used for on-column DNase I treatment. After elution in

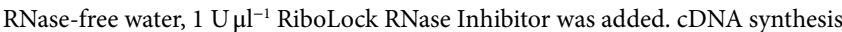
was performed using random nonamer primers (Gene Link, pd(N)9, 26-400006, final concentration: $5 \mu \mathrm{M}$ ) and the SuperScript III Reverse Transcriptase (Thermo Fisher Scientific) according to the manufacturer's instructions using $2 \mu \mathrm{g}$ total RNA. cDNA was generated using the GeneAmp PCR System 9700 (Applied Biosystems; cycle: $25^{\circ} \mathrm{C}$ for $5 \mathrm{~min}, 50^{\circ} \mathrm{C}$ for $60 \mathrm{~min}, 70^{\circ} \mathrm{C}$ for $15 \mathrm{~min}$, $4^{\circ} \mathrm{C}$ hold). DNA oligonucleotides (Sigma-Aldrich) for the genes of interest (GOIs) and the reference gene Ywhaz (tyrosine 3-monooxygenase/tryptophan 5 -monooxygenase activation protein, zeta $)^{42}$. The following primer sequences were used for qRT-PCR: Ywhaz forward 5' -CACGCTCCCTAACCTTGCTT-3 , reverse 5'-ATCGTAGAAGCCTGACGTGG-3'

; Grb10 forward 5'-AAGCGAAGACCGAGATGAAG-3'

, reverse 5'-CATAGGTGCGTTGAAAGGAG-3'

; Igf2 forward 5'-CTTGGATCCCAGAACCCAAGAA-3

, reverse 5' -CCCCTTGGTGACATGGGGAC-3'

- Slc38a1 forward 5'-CGGGAGAGTAGGAGGAGTCT-3'

, reverse 5'-GTCTGCTCCCACACATCGTT-3'; Slc38a2

forward 5'-AATGCGATTGTGGGCAGTGG-3', reverse 5'

-AGCTTTCCAGCCAGACCATAC-3'. In brief, singleplex reactions $(20 \mu \mathrm{l})$ were set up manually in PCR grade water (Roche) in LightCycler 480 Multiwell Plate 96 Reaction Plate (Roche Life Science): $10 \mu$ l FastStart Essential DNA Green Master (Roche Life Science); $300 \mathrm{nM}$ of forward and reverse primer each; and $2 \mu$ 1 cDNA template. qRT-PCR runs were conducted on the LightCycler 96 system (Roche Life Science) with end point fluorescence detection: $10 \mathrm{~min}$ at $95^{\circ} \mathrm{C}$ and 50 amplification cycles $\left(15 \mathrm{~s}\right.$ at $95^{\circ} \mathrm{C}, 10 \mathrm{~s}$ at $65^{\circ} \mathrm{C}$ and $20 \mathrm{~s}$ at $\left.72^{\circ} \mathrm{C}\right)$. Analysis was performed in triplicate for each GOI and Ywhaz in each sample. Negative controls and sample without reverse transcriptase were included to detect possible contaminations. Relative expression values were determined using the $\mathrm{e}^{-\Delta \Delta C_{\mathrm{t}}}$ method $^{42}$. The Rn values were exported from the LightCycler 96 software (Roche Life Science) to Microsoft Office Excel and $N_{0}$ values for the starting concentration of the transcript in the original sample were obtained with the LinRegPCR software version 11.160. The averaged $N_{0}$ value of the GOI ( $n=3$ technical replicates) was then normalized to the averaged $N_{0}$ value for $Y w h a z\left(N_{0(\text { Ywhaz }}\right)$ of the respective sample. The relative $N_{0(\mathrm{GOI})} / N_{0(\mathrm{Ywhaz})}$ expression values of the biological replicates are presented. The relative $N_{0(\mathrm{GOI})} / N_{0 \text { (Ywhaz) }}$ expression of PBS was set to 1 for each gene.

Immunohistochemistry of fetal tissues. Sagittal sections of fetal heads, including brains, were cut without decalcification for haematoxylin and eosin (H\&E) evaluation, terminal deoxynucleotidyl transferase dUTP nick end labelling (TUNEL) assay staining and ionised calcium-binding adapter molecule 1 (Iba1) immunostaining. Sagittal sections from perfused 4-week-old offspring brains were cut, with IgM-specific antibody (Thermo Fisher Scientific) and 4,6-diamidino2-phenylindole (DAPI; PanReac AppliChem) as nuclear counterstaining. In the pregnant uterus of ZIKV-infected and control mice, the presence of necrosis, haemorrhages, inflammation, as well as a blastocyst/embryo and invasion of epithelial cells, was determined for each placentation site on $\mathrm{H} \& \mathrm{E}$ and cytokeratinstained slides (polyclonal rabbit anti-cytokeratin, Dako; 1:500; pretreatment: $20 \mathrm{~min}$ microwave in citrate buffer, $\mathrm{pH}=6.0$ ), respectively. TUNEL assay and cytokeratin immunohistochemistry were performed as described previously ${ }^{1,43}$ All TUNEL-positive cells in an area of $0.25 \mu \mathrm{m}^{2}$ were counted within the brainstem of ZIKV-infected and control mice. Primary antibodies against Ibal (1:1500; Wako Chemicals) were visualized using the avidin-biotin complex method with 3,3' -diaminobenzidine as the substrate. Slides were analysed using a NanoZoomer 2.0-RS digital slide scanner and NDP.view2 software (both Hamamatsu). Microglia cells were defined by morphology and counted manually with the help of the ImageJ software. The eyes from offspring were dehydrated in an ascending series of sucrose, embedded in Tissue-Tek (Sakura Finetek) and sectioned at a thickness of $25 \mu \mathrm{m}$. Central (that is, in the plane of the optic disc) retina sections were selected, blocked for $1 \mathrm{~h}$ in PBS containing $0.1 \%$ bovine serum albumin and $0.3 \%$ Triton X-100 (both Sigma-Aldrich) and incubated with anti-glial fibrillary acidic protein (GFAP) antibodies (Dako) to stain astrocytes, anti-Ibal (Wako Chemicals) to stain microglia cells, anti-recoverin antibodies (Merck Millipore) to stain rod and cone photoreceptor cells and biotinylated peanut agglutinin (PNA; Vector Laboratories) to stain cone photoreceptor cells. Primary antibodies and biotinylated PNA were detected with Cy3-conjugated secondary antibodies and Cy3-conjugated streptavidin (Jackson ImmunoResearch) respectively, and sections were stained with DAPI (Sigma-Aldrich) and mounted onto slides. For each antigen, sections of eight offspring from ZIKV-infected and eight offspring from control dams were coded and analysed in a blinded manner. Photomicrographs are $z$ projections of image stacks through the entire thickness of the sections and were taken from retina regions close to the optic disc using an Axio Observer.Z1 microscope with ApoTome.2 (ZEISS).

Golgi staining. Whole brains were harvested from the 8-week-old offspring of ZIKV-infected and control dams and stained using the FD Rapid GolgiStain Kit (FD NeuroTechnologies). In brief, brains were rinsed with double-distilled water and then immersed in a 1:1 mixture of FD Solution A:B. Solution A:B was replaced within the first $24 \mathrm{~h}$ and then kept for 2 weeks at room temperature in the dark. Brains were then transferred to FD Solution $\mathrm{C}$ and kept in the dark at $4{ }^{\circ} \mathrm{C}$ for $48 \mathrm{~h}$. Solution C was replaced after the first $24 \mathrm{~h}$. Afterwards, in preparation for vibratome sectioning, individual brains were placed in sterile $30 \%$ sucrose at $4{ }^{\circ} \mathrm{C}$ for $24 \mathrm{~h}$. Coronal sections of $120 \mu \mathrm{m}$ thickness were cut and collected consecutively in a 24-well plate in PBS. This thickness enabled optimal staining and preservation of spines on secondary and tertiary dendritic segments, while the complete basal dendritic arbor was acquired as needed for Sholl analysis. Sections were mounted on SuperFrost Ultra Plus microscope slides (Thermo Fisher Scientific) and allowed to dry for 5-10 min. Sections were then developed exactly as described in the FD Rapid GolgiStain instructions. The development solution should be prepared just before use combining FD Solution D:E:double-distilled water in a 1:1:2 mixture. Glass slides were immersed for 3-5 min, rinsed in double-distilled water twice ( $4 \mathrm{~min}$ each) and allowed to dry briefly. Each slide was embedded using 120-150 $\mu \mathrm{l}$ Mowiol (Sigma-Aldrich). Two-to-three independent coronal sections per mouse, which contained the dorsal hippocampus (bregma: -1.22 to $-2.06 \mathrm{~mm}$; interaural: 2.58 to $1.74 \mathrm{~mm})^{44}$ were imaged. CA1 pyramidal neurons were identified by localization within the hippocampus and because of their distinct morphology. Presence of a completely unclipped basal or apical dendritic arbor was a prerequisite for selection and imaging of the cell. $z$ stacks of Golgistained dendritic trees (60-90 $\mu \mathrm{m}$ total on the $z$ axis; optical section thickness per $z$ section: $1 \mu \mathrm{m}$ ) were taken at $\times 60$ magnification on a Nikon Eclipse Ti-E inverted microscope fitted with a CoolSNAP HQ2 CCD camera (Photometrics).

Golgi spine analysis. $Z$ stacks were opened in ImageJ. The image was overlaid with 4 concentric circles $(20 \mu \mathrm{m}$ distance each) using the ROI manager tool to define medial $\left(2^{\circ}, 30-50 \mu \mathrm{m}\right.$ distance to soma centre $)$ and distal $\left(3^{\circ}, 50-70 \mu \mathrm{m}\right.$ distance to soma centre) dendritic segments. For apical dendrites, the same procedure was followed but with branching points at the main dendrite as the focal point. Spine analysis was performed on selected dendritic segments according to standardized parameters described previously ${ }^{45}$ using the ImageJ selection tools combined with the ROI manager. In short, to sort spines into categories, spine head width 
and length were considered as well as their ratio. Numbers of analysed spines per segment were then converted into densities.

Sholl analysis. To perform the Sholl analysis, basal dendritic arbors of pyramidal cells of CA1 were traced with the ImageJ segmented line tool to create a twodimensional image of the basal dendritic tree. This image was then analysed with the Sholl analysis plugin with the radii set to $5 \mu \mathrm{m}$ intervals ${ }^{46}$.

Heat maps and assessment of dendritic coverage. Heat maps were generated to create a visual representation using ImageJ on the basis of the dendritic filaments of individual cells. The dendritic width of these filaments was reduced to $1 \mathrm{px}$, thus taking each dendritic section equally into account. Neurons were matched in their orientation and aligned (angular position of apical dendrite; centroid of soma). Subsequently, filaments were projected onto each other followed by the application of a mean filter (radius $=5 \mathrm{px}$ ). To put an emphasis on areas with a higher occurrence of dendritic material, a look-up table (warm metal) was applied. To assess dendritic coverage within the dendritic field, coordinates of non-background pixels were extracted from the images of the dendritic filament and evaluated regarding their angular position. Therefore, the centroid of the soma was used as a reference, as well as a vector marking $0^{\circ}$. Applying equation (1), the angular distribution was assessed, correcting for angles $>180^{\circ}$. These values were then divided into $30^{\circ}$ bins with the result depicted as the average percentage dendritic material per cell in a customized rose plot generated with Excel.

$$
\cos (\alpha)=\frac{\mathbf{a} * \mathbf{b}}{|\mathbf{a}| *|\mathbf{b}|}, 0^{\circ}<\alpha<180^{\circ}
$$

Open field analysis. The open field analysis was carried out during the first half of the light phase and as described previously ${ }^{47}$. It consisted of a transparent and infrared light-permeable acrylic test arena with a smooth floor (internal measurements: $45.5 \times 45.5 \times 39.5 \mathrm{~cm}^{3}$ ). Illumination levels were set at approximately $150 \mathrm{~lx}$ in the corners and 200 lx in the middle of the test arena. Data were recorded and analysed using the ActiMot system (TSE Systems).

Y-maze. Spontaneous alternation in the Y-maze was tested during the first half of the light phase and as described previously ${ }^{48}$. The apparatus was made of opaque light grey polyvinyl chloride and had three identical arms $\left(30 \times 5 \times 15 \mathrm{~cm}^{3}\right)$ placed at $120^{\circ}$ from each other; illumination in the centre of the maze was $100 \mathrm{~lx}$. Each mouse was placed at the end of one arm and allowed to move freely through the maze during a 5-min session. Spontaneous alternations (defined as consecutive entries into all three arms without repetitions in overlapping triplet sets) were scored. The total numbers of arm entries were collected cumulatively over the $5 \mathrm{~min}$. Spontaneous alternation performance percentage is defined as the ratio of actual (total alternations) to possible alternations (total number of triplets) $\times 100$. When placed in the Y-maze as a novel environment, normal mice prefer to explore the least recently visited arm, and thus tend to alternate visits between the three arms. Therefore, alternation behaviour is a measure of spatial working memory since the mouse must maintain an ongoing record of the most recently visited arms to explore the three arms successively, and continuously update such records.

Object recognition. The object-recognition test was carried out during the light phase and as described previously ${ }^{48}$. In brief, each mouse was allowed to explore two identical objects three times for $5 \mathrm{~min}$, with an inter-trial interval of $15 \mathrm{~min}$. After a retention interval of $3 \mathrm{~h}$, one of the previously encountered familiar objects was substituted by a new, unfamiliar one, which was again substituted by a novel object after a second retention interval of $24 \mathrm{~h}$. For each retention test, the mouse was put again into the test box for $5 \mathrm{~min}$ and exploration time-defined as touching the object with the nose-was recorded by a trained observer with a handheld device. An object recognition index was calculated as the time spent investigating the unfamiliar object divided by the time spent investigating both the familiar and unfamiliar objects.

Virtual drum. A virtual optomotor response (OMR) system (OptoMotry System; CerebralMechanics) was used to test each animal's visual function as described previously ${ }^{49,50}$. In brief, the mouse was placed on a platform surrounded by a virtual rotating cylinder formed by four computer monitors that displayed rotating vertical sine wave gratings and was observed by a trained human experimenter. Positive reflexive head tracking movements in the direction of the grating rotation were used to determine spatial frequency thresholds. To determine spatial frequency thresholds, grating contrast was held at $100 \%$ while spatial frequency was increased in a staircase paradigm until the response threshold was crossed ${ }^{49}$. All OMR stimulation was done at approximately the same time daily during the first half of the light phase for the duration of the experiment, with each testing session lasting about $10 \mathrm{~min}$.

Morris water maze. The MWM test was performed during the light phase (water tank illuminated with white light (45lx on average) as described previously $)^{51}$ Following a habituation day (day 0 ), during which mice were placed on the submerged platform three times for $10 \mathrm{~s}$ to familiarize the animals with the test apparatus (a black circular plastic tank filled with water $\left(24^{\circ} \mathrm{C}\right)$ coloured opaque with milk (to make the black mice visible for the camera and software) with a submerged (hidden) black platform and external visual cues), place learning was performed over ten consecutive days with four 1-min trials per day. Each day, the animals were put into the pool at four different starting points; the starting point order in the four quadrants of the tank was changed from day to day, whereas the hidden platform stayed in the same quadrant of the maze throughout the testing period. For each trial, swim velocity, distance moved and escape latency were measured by a computerized tracking system (EthoVision). For each of the 1,280 individual trials of this experiment, the search strategy used during navigation in the MWM in an individual trial was categorized into spatial or non-spatial as described previously ${ }^{33}$.

Phylogeny. The phylogenetic tree was inferred based on the complete genome sequences using the maximum likelihood method in PhyML 3.0 $0^{52}$ with 1,000 pseudoreplicates; in parallel, the Bayesian Markov chain Monte Carlo treesampling method-by using MrBayes 3.1.2. genome sequences of the isolates from this study and those retrieved from GenBank-were aligned using CLUSTALW in Geneious 9.1.5. Potential recombinant strains have been removed from the phylogenetic analysis. The Akaike information criterion was chosen as the model selection framework and the general time-reversible model of sequence evolution with gamma-distributed rate variation among sites $(\mathrm{GTR}+\Gamma)$ as the best model. Statistical support of grouping from bootstrap replicates $(\geq 70 \%)$ and Bayesian posterior probabilities (clade credibility $\geq 90 \%$ ) is indicated with an asterisk.

Magnetic resonance imaging.. For the ex vivo magnetic resonance imaging (MRI) examination, the heads of five offspring from ZIKV-infected and control dams were placed in $50 \mathrm{ml}$ Falcon tubes filled with $4 \%$ formalin. Measurements were performed with a preclinical high-field scanner (7 T ClinScan; Bruker Biospin) equipped with a $660 \mathrm{mT} \mathrm{m}^{-1}$ gradient coil. Tubes were placed in a circular polarized $1 \mathrm{H}$ mouse whole body radiofrequency coil. A three-dimensional T2-weighted turbo spin-echo sequence with 40 slices of $200 \mu \mathrm{m}$ thickness was planned axial to cover the brain. The field of view was $32 \mathrm{~mm}$ with a matrix of $320 \times 320$ px resulting in a voxel size of $100 \times 100 \times 200 \mu^{3}$. Further sequence parameters were TE (echo time) $23 \mathrm{~ms}$, repetition time $2500 \mathrm{~ms}$, turbo factor of 11 and number of average $=1$. Exported DICOM (Digital Imaging and Communications in Medicine) images were analysed with ImageJ.

\section{Patient recruitment, sample collection and sample processing. Pregnant} women (aged 14-26) (gestation third trimester) were recruited based on clinical signs and symptoms (rash, muscle aches, eye pain, headache) for ZIKV or related flavivirus infections to take part in the longitudinal observational clinical study at the Hospital Regional Iquitos in Iquitos, Peru in 2017. Blood samples were collected from pregnant women by venepuncture as part of their routine examination and processed to serum using standard clinical laboratory protocols. Serum samples were subjected to qRT-PCR against ZIKV at the US Naval Medical Research Center No. 6 (NAMRU-6) in Lima, Peru. Depending on qRT-PCR outcome, patient samples were defined as ZIKV RNA-positive or ZIKV RNAnegative mothers. One aliquot per pregnant woman was immediately frozen after processing to serum and stored until shipping at $-80^{\circ} \mathrm{C}$. Both patient groups were recruited back post-partum and blood samples from newborns (within their first month of life) were collected during routine paediatric examination and processed to serum using standard clinical laboratory protocols. None of the newborns was diagnosed with microcephaly or other clinically apparent phenotypes. Serum samples from newborns were subjected to qRT-PCR against ZIKV performed at NAMRU-6. One aliquot per newborn was immediately frozen after processing to serum and stored until shipping at $-80^{\circ} \mathrm{C}$. All frozen serum samples taken from mothers and children were then shipped on dry ice to the Heinrich Pette Institute, Hamburg, Germany for the detection of TST levels.

The study protocol was reviewed and approved by the Institutional Review Board of the Tropical Pathology and Infectious Disease Association (Cusco, Peru) with the study code IPTEI-2016/003. Eligible pregnant women were informed about the study purpose and ethical consent was obtained from the study participants. Newborns were enrolled based on the consent provided by their mothers.

Data analysis. Animal size estimates were calculated depending on the scientific question raised either by a Kruskal-Wallis one-way analysis of variance (ANOVA) or a Mann-Whitney $U$-test. No statistical methods were used to predetermine sample size regarding the in vitro experiments. Experiments were randomized and investigators were blinded to data assessment of MWM and retina morphology/ pathology. In the German Mouse Clinic, a certified mouse phenotype assessment centre, experiments are conducted in a counterbalanced design to account for circadian effects (not blinded) and routinely performed by trained experimenters according to standard operating procedures. All data were analysed with the Prism software (GraphPad) using a two-way ANOVA followed by Bonferroni post hoc correction or Šidák's multiple comparison, Fisher's exact test or Student's $t$-test as indicated in the respective legends. Behavioural data were analysed with two-way 
ANOVAs (factors: sex $\times$ maternal treatment) or one-way ANOVAs (PBS, high and storm TST level groups) when appropriate. Data from the MWM were analysed with ANOVA followed by Fisher's least significant difference test. The frequencies of search strategies were analysed with Barnard's test.

Reporting Summary. Further information on research design is available in the Nature Research Reporting Summary linked to this article.

\section{Data availability}

Accession numbers of the ZIKV strains used to perform the phylogenetic analysis are indicated in Supplementary Fig. 1. The data that support the findings of this study are available from the corresponding author on request.

Received: 28 March 2018; Accepted: 1 August 2018; Published online: 10 September 2018

\section{References}

1. Dick, G. W., Kitchen, S. F. \& Haddow, A. J. Zika virus. I. Isolations and serological specificity. Trans. R. Soc. Trop. Med. Hyg. 46, 509-520 (1952).

2. Driggers, R. W. et al. Zika virus infection with prolonged maternal viremia and fetal brain abnormalities. N. Engl. J. Med. 374, 2142-2151 (2016).

3. Faria, N. R. et al. Zika virus in the Americas: early epidemiological and genetic findings. Science 352, 345-349 (2016)

4. França, G. V. et al. Congenital Zika virus syndrome in Brazil: a case series of the first 1501 livebirths with complete investigation. Lancet 388, 891-897 (2016).

5. van der Eijk, A. A. Miscarriage associated with Zika virus infection. N. Engl. J. Med. 375, 1002-1004 (2016).

6. Brasil, P. et al. Zika virus infection in pregnant women in Rio de Janeiro. N. Engl. J. Med. 375, 2321-2334 (2016).

7. Cauchemez, S. et al. Association between Zika virus and microcephaly in French Polynesia, 2013-15: a retrospective study. Lancet 387, 2125-2132 (2016).

8. Kapogiannis, B. G., Chakhtoura, N., Hazra, R. \& Spong, C. Y. Bridging knowledge gaps to understand how Zika virus exposure and infection affect child development. JAMA Pediatr. 171, 478-485 (2017).

9. Cugola, F. R. et al. The Brazilian Zika virus strain causes birth defects in experimental models. Nature 534, 267-271 (2016).

10. Miner, J. J. et al. Zika virus infection during pregnancy in mice causes placental damage and fetal demise. Cell 165, 1081-1091 (2016).

11. $\mathrm{Li}, \mathrm{C}$. et al. Zika virus disrupts neural progenitor development and leads to microcephaly in mice. Cell Stem Cell 19, 120-126 (2016).

12. Yockey, L. J. et al. Vaginal exposure to Zika virus during pregnancy leads to fetal brain infection. Cell 166, 1247-1256 (2016).

13. Engels, G. et al. Pregnancy-related immune adaptation promotes the emergence of highly virulent $\mathrm{H} 1 \mathrm{~N} 1$ influenza virus strains in allogenically pregnant mice. Cell Host Microbe 21, 321-333 (2017).

14. Shin Yim, Y. et al. Reversing behavioural abnormalities in mice exposed to maternal inflammation. Nature 549, 482-487 (2017)

15. Estes, M. L. \& McAllister, A. K. Maternal immune activation: implications for neuropsychiatric disorders. Science 353, 772-777 (2016).

16. Leuner, B. \& Shors, T. J. New spines, new memories. Mol. Neurobiol. 29 , 117-130 (2004).

17. Maliqueo, M., Echiburú, B. \& Crisosto, N. Sex steroids modulate uterineplacental vasculature: implications for obstetrics and neonatal outcomes. Front. Physiol. 7, 152 (2016).

18. Shors, T. J. \& Miesegaes, G. Testosterone in utero and at birth dictates how stressful experience will affect learning in adulthood. Proc. Natl Acad. Sci. USA 99, 13955-13960 (2002).

19. Barbazanges, A., Piazza, P. V., Le Moal, M. \& Maccari, S. Maternal glucocorticoid secretion mediates long-term effects of prenatal stress. J. Neurosci. 16, 3943-3949 (1996)

20. Li, H. et al. Zika virus infects neural progenitors in the adult mouse brain and alters proliferation. Cell Stem Cell 19, 593-598 (2016).

21. Coan, P. M. et al. Adaptations in placental nutrient transfer capacity to meet fetal growth demands depend on placental size in mice. J. Physiol. 586, 4567-4576 (2008).

22. He, S., Allen, J. C. Jr., Malhotra, R., Østbye, T. \& Tan, T. C. Association of maternal serum progesterone in early pregnancy with low birth weight and other adverse pregnancy outcomes. J. Matern. Fetal Neonatal Med. 29, 1999-2004 (2016).

23. Aiken, C. E. \& Ozanne, S. E. Sex differences in developmental programming models. Reproduction 145, R1-R13 (2013).

24. Manikkam, M. et al. Fetal programming: prenatal testosterone excess leads to fetal growth retardation and postnatal catch-up growth in sheep. Endocrinology 145, 790-798 (2004).

25. Meikle, D. B. \& Drickamer, L. C. Food availability and secondary sex ratio variation in wild and laboratory house mice (Mus musculus). J. Reprod. Fertil. 78, 587-591 (1986).
26. Nguyen, T. V. et al. Sex-specific associations of testosterone with prefrontalhippocampal development and executive function. Psychoneuroendocrinology 76, 206-217 (2017).

27. Miyamoto, A. et al. Microglia contact induces synapse formation in developing somatosensory cortex. Nat. Commun. 7, 12540 (2016)

28. Miner, J. J. et al. Zika virus infection in mice causes panuveitis with shedding of virus in tears. Cell Rep. 16, 3208-3218 (2016).

29. van den Pol, A. N., Mao, G., Yang, Y., Ornaghi, S. \& Davis, J. N. Zika virus targeting in the developing brain. J. Neurosci. 37, 2161-2175 2017).

30. Anacker, C. \& Hen, R. Adult hippocampal neurogenesis and cognitive flexibility-linking memory and mood. Nat. Rev. Neurosci. 18, 335-346 (2017)

31. D'Hooge, R. \& De Deyn, P. P. Applications of the Morris water maze in the study of learning and memory. Brain Res. Rev. 36, 60-90 (2001)

32. Janus, C. Search strategies used by APP transgenic mice during navigation in the Morris water maze. Learn. Mem. 11, 337-346 (2004).

33. Brody, D. L. \& Holtzman, D. M. Morris water maze search strategy analysis in PDAPP mice before and after experimental traumatic brain injury. Exp. Neurol. 197, 330-340 (2006).

34. Lee, A. S., Duman, R. S. \& Pittenger, C. A double dissociation revealing bidirectional competition between striatum and hippocampus during learning. Proc. Natl Acad. Sci. USA 105, 17163-17168 (2008).

35. Sabuncu, M. R. et al. Morphometricity as a measure of the neuroanatomical signature of a trait. Proc. Natl Acad. Sci. USA 113, E5749-E5756 (2016).

36. Celec, P., Ostatníková, D. \& Hodosy, J. On the effects of testosterone on brain behavioral functions. Front. Neurosci. 9, 12 (2015).

37. Adams Waldorf, K. M. et al. Congenital Zika virus infection as a silent pathology with loss of neurogenic output in the fetal brain. Nat. Med. 24, 368-374 (2018)

38. Russell, P. K., Udomsakdi, S. \& Halstead, S. B. Antibody response in dengue and dengue hemorrhagic fever. Jpn. J. Med. Sci. Biol. 20, 103-108 (1967).

39. Styer, L. M. et al. Mosquitoes inoculate high doses of West Nile virus as they probe and feed on live hosts. PLoS Pathog. 3, 1262-1270 (2007).

40. Croy, B. A., Yamada, A. T., DeMayo, F. J. \& Adamson, S. L. The Guide to Investigation of Mouse Pregnancy (Academic, San Diego, CA, 2014).

41. Tappe, D. et al. Ross River virus infection in a traveller returning from northern Australia. Med. Microbiol. Immunol. 198, 271-273 (2009).

42. Solano, M. E., Thiele, K., Kowal, M. K. \& Arck, P. C. Identification of suitable reference genes in the mouse placenta. Placenta 39, 7-15 (2016).

43. Mu, J., Slevin, J. C., Qu, D., McCormick, S. \& Adamson, S. L. In vivo quantification of embryonic and placental growth during gestation in mice using micro-ultrasound. Reprod. Biol. Endocrinol. 6, 34 (2008).

44. Paxinos, G. \& Franklin, K. B. J. Paxinos and Franklin's the Mouse Brain in Stereotaxic Coordinates 4th edn (Elsevier, Amsterdam, 2012).

45. Risher, W. C., Ustunkaya, T., Singh Alvarado, J. \& Eroglu, C. Rapid Golgi analysis method for efficient and unbiased classification of dendritic spines. PLoS ONE 9, el07591 (2014).

46. Ferreira, T. A. et al. Neuronal morphometry directly from bitmap images. Nat. Methods 11, 982-984 (2014).

47. Hölter, S. M. et al. Tests for anxiety-related behavior in mice. Curr. Protoc. Mouse Biol. 5, 291-309 (2015).

48. Hölter, S. M. et al. Assessing cognition in mice. Curr. Protoc. Mouse Biol. 5, 331-358 (2015).

49. Mui, A. M. et al. Daily visual stimulation in the critical period enhances multiple aspects of vision through BDNF-mediated pathways in the mouse retina. PLoS ONE 13, e0192435 (2018).

50. Prusky, G. T., Alam, N. M., Beekman, S. \& Douglas, R. M. Rapid quantification of adult and developing mouse spatial vision using a virtual optomotor system. Invest. Ophthalmol. Vis. Sci. 45, 4611-4616 (2004).

51. Gröticke, I., Hoffmann, K. \& Löscher, W. Behavioral alterations in a mouse model of temporal lobe epilepsy induced by intrahippocampal injection of kainate. Exp. Neurol. 213, 71-83 (2008).

52. Guindon, S. et al. New algorithms and methods to estimate maximumlikelihood phylogenies: assessing the performance of PhyML 3.0. Syst. Biol. 59, 307-321 (2010).

\section{Acknowledgements}

The Heinrich Pette Institute, Leibniz Institute for Experimental Virology is supported by the Free and Hanseatic City of Hamburg and the Federal Ministry of Health. This study was supported by the Federal Ministry of Health (G.G.), the German Research Center for Infection (DZIF) (G.G.), the Niedersachsen-Research Network on Neuroinfectiology (N-RENNT) of the Ministry of Science and Culture of Lower Saxony, Germany (W.B., W.L.) and the German Federal Ministry of Education and Research (Infrafrontier grant 01KX1012) (M.H.A.). F.C.d.A. is supported by Deutsche Forschungsgemeinschaft (DFG) Grant (FOR 2419, CA1495/1-1 and CA 1495/4-1), ERA-NET Neuron Grant (Bundesministerium für Bildung und Forschung, BMBF, 01EW1410 ZMNH AN B1), Landesforschungsförderung Hamburg (Z-AN LF) and University Medical Center Hamburg-Eppendorf (UKE). R.B. is supported by the Deutsche Forschungsgemeinschaft (BA 1505/8-1). We are grateful for the excellent technical contribution of all staff at 
the technology platform small animal models of the Heinrich Pette Institute, Leibniz Institute for Experimental Virology, Hamburg and the technical staff of the Department of Pathology, University of Veterinary Medicine, Hannover. We thank T. Andreas from the Department of Obstetrics and Fetal Medicine at the University Medical Center Hamburg-Eppendorf for his excellent technical support with the preparation of pregnant mice. We thank P. Pruunsild and H. Bading, Department of Neurobiology, Interdisciplinary Center for Neurosciences at Heidelberg University for providing human NPCs. We thank U. Markert, Department of Obstetrics, Placenta-Lab, University Hospital Jena for providing the human placental cells.

\section{Author contributions}

G.G. conceived the study. S.S.-B. and G.G. designed and coordinated the experiments. S.S.-B., K.W.-G., C.D., A.P., G.P.-S., U.M. and S.Thi. performed all infection studies in mice. TST treatment of mice was performed by H.L., J.S. and S.H. S.Tha., I.A.A., T.S., N.M.K., C.D. and K.W.-G. performed the qRT-PCR assays. S.Tha., C.D., K.W.-G. and A.P. performed the hormone ELISA as well as the cytokine assays. T.R. measured the TST levels. V.H., W.B., V.M.P. and I.G. performed the histological and immunohistological examinations, TUNEL and cytokeratin staining, and the in situ hybridization and analysis. B.S. performed the Iba1 staining and respective analysis of fetal brains. H.I. performed the MRI scans on the brains of the offspring. M.R. performed Golgi staining and assisted with spine analysis. R.S. performed image acquisition, and the dendritic and spine analyses. F.C.d.A. coordinated the brain analysis. U.B. performed the immunohistochemical analyses of retinas. T.M. and R.B. performed the ZIKV replication kinetics in cell culture. Histopathological findings were analysed and discussed by P.A., M.A.F., V.H., I.G., V.M.P. and W.B. S.J. and T.S. measured viral IgG and IgM titres. D.C. performed the phylogenetic analysis. S.M.H., O.A., F.M., V.K., R.D., L.S., W.L., I.W. and C.K. performed and/or analysed the behavioural experiments. H.F. and M.H.d.A. coordinated and conceived the animal phenotypic tests. S.B. and L.R. were responsible for the recruitment of the mother-child cohort in Iquitos, Peru and for the qRT-PCR analysis of patient sera. J.S.-C., O.V. and M.G. provided material, analysed the data and discussed the study. S.S.-B. and G.G. wrote the manuscript. All authors revised the manuscript.

\section{Competing interests}

The authors declare no competing interests.

\section{Additional information}

Supplementary information is available for this paper at https://doi.org/10.1038/ s41564-018-0236-1.

Reprints and permissions information is available at www.nature.com/reprints. Correspondence and requests for materials should be addressed to G.G.

Publisher's note: Springer Nature remains neutral with regard to jurisdictional claims in published maps and institutional affiliations. 


\section{Reporting Summary}

Nature Research wishes to improve the reproducibility of the work that we publish. This form provides structure for consistency and transparency in reporting. For further information on Nature Research policies, see Authors \& Referees and the Editorial Policy Checklist.

\section{Statistical parameters}

When statistical analyses are reported, confirm that the following items are present in the relevant location (e.g. figure legend, table legend, main text, or Methods section).

$\mathrm{n} / \mathrm{a} \mid$ Confirmed

$\square$ The exact sample size $(n)$ for each experimental group/condition, given as a discrete number and unit of measurement

$\square$ \ An indication of whether measurements were taken from distinct samples or whether the same sample was measured repeatedly

$\square$ The statistical test(s) used AND whether they are one- or two-sided

Only common tests should be described solely by name; describe more complex techniques in the Methods section.

$\square$ A description of all covariates tested

$\square$ Х A description of any assumptions or corrections, such as tests of normality and adjustment for multiple comparisons

$\square$ A full description of the statistics including central tendency (e.g. means) or other basic estimates (e.g. regression coefficient) AND

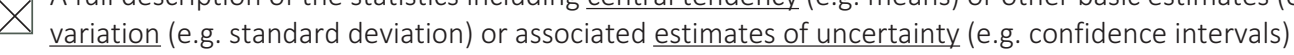

$\square$ For null hypothesis testing, the test statistic (e.g. $F, t, r$ ) with confidence intervals, effect sizes, degrees of freedom and $P$ value noted

Give P values as exact values whenever suitable.

Х $\square$ For Bayesian analysis, information on the choice of priors and Markov chain Monte Carlo settings

Х $\square$ For hierarchical and complex designs, identification of the appropriate level for tests and full reporting of outcomes

$\bigotimes \square$ Estimates of effect sizes (e.g. Cohen's $d$, Pearson's $r$ ), indicating how they were calculated

$\varnothing$ Clearly defined error bars

State explicitly what error bars represent (e.g. SD, SE, CI)

Our web collection on statistics for biologists may be useful.

\section{Software and code}

Policy information about availability of computer code

Data collection n/a

Data analysis

$\mathrm{n} / \mathrm{a}$

For manuscripts utilizing custom algorithms or software that are central to the research but not yet described in published literature, software must be made available to editors/reviewers upon request. We strongly encourage code deposition in a community repository (e.g. GitHub). See the Nature Research guidelines for submitting code \& software for further information.

\section{Data}

Policy information about availability of data

All manuscripts must include a data availability statement. This statement should provide the following information, where applicable:

- Accession codes, unique identifiers, or web links for publicly available datasets

- A list of figures that have associated raw data

- A description of any restrictions on data availability

A full data availability statement is included in the manuscript. 


\section{Field-specific reporting}

Please select the best fit for your research. If you are not sure, read the appropriate sections before making your selection.

$\bigotimes$ Life sciences $\quad \square$ Behavioural \& social sciences $\quad \square$ Ecological, evolutionary \& environmental sciences

For a reference copy of the document with all sections, see nature.com/authors/policies/ReportingSummary-flat.pdf

\section{Life sciences study design}

All studies must disclose on these points even when the disclosure is negative.

Sample size Kruskall-Wallis one-way ANOVA or Wilcoxon-Mann-Whitney Test

Data exclusions All obtained data were included unless specifically stated, such as on page 12 according to pre-established inclusion/exclusion criteria.

Replication All experimental findings were realiably reproducable.

Randomization Samples were coded and analyzed in a blinded manner (page 16)

Blinding Investigators were blinded for data collection and analysis in terms of histological sections.

\section{Reporting for specific materials, systems and methods}

Materials \& experimental systems

$\mathrm{n} / \mathrm{a}$ Involved in the study

$\searrow \square$ Unique biological materials

$\square$ Х Antibodies

$\square$ Х Eukaryotic cell lines

\ $\square$ Palaeontology

$\bigotimes$ Animals and other organisms

$\square$ \Human research participants

\author{
Methods \\ $\mathrm{n} / \mathrm{a}$ Involved in the study \\ \ $\square$ ChIP-seq \\ Х $\square$ Flow cytometry \\ $\square$ Х MRI-based neuroimaging
}

\section{Antibodies}

Antibodies used

Validation
All information are provided in text, see page 13-16

All antibodies used were tested with appropriate negative and positive control samples.

\section{Eukaryotic cell lines}

\section{Policy information about cell lines}

Cell line source(s)

All information are provided in text, see page 11

Authentication

None of the cell lines used have been authenticated.

Mycoplasma contamination

All information are provided in text, see page 11

Commonly misidentified lines

(See ICLAC register)

No commonly misidentified cell lines were used.

\section{Animals and other organisms}

Policy information about studies involving animals; ARRIVE guidelines recommended for reporting animal research 
Wild animals

Field-collected samples

\section{Human research participants}

Policy information about studies involving human research participants

Population characteristics All information are provided in text, see page 20-21

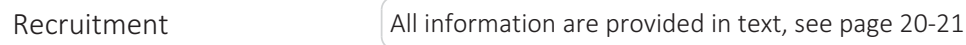

\section{Magnetic resonance imaging}

Experimental design

Design type

Design specifications

Behavioral performance measures

\section{Acquisition}

Imaging type(s)

Field strength

Sequence \& imaging parameters

Area of acquisition

Diffusion MRI Used

Preprocessing

Preprocessing software

Normalization

Normalization template

Noise and artifact removal

Volume censoring

Statistical modeling \& inference

Model type and settings

Effect(s) tested

no model types used

not used

Specify type of analysis: $\bigotimes$ Whole brain

$\square$ ROI-based

Exported DICOM images were viewed and compared offline with the image processing software ImageJ (National Institute of Health, Maryland, USA).

not used

not used

not used

not used

Statistic type for inference

(See Eklund et al. 2016)

Correction

not used

not used

Models \& analysis

$\mathrm{n} / \mathrm{a}$ Involved in the study

Х $\square$ Functional and/or effective connectivity

Х $\square$ Graph analysis

Х $\square$ Multivariate modeling or predictive analysis 